Pacific Journal of Mathematics

THE BIDUAL OF A LOCALLY MULTIPLICATIVELY-CONVEX SidNey (DenNy) L. GULICK 


\title{
THE BIDUAL OF A LOCALLY MULTIPLICATIVELY-CONVEX ALGEBRA
}

\author{
S. L. GuLICK
}

This paper combines two extensions of the theory of Banach algebras. On the one hand, Arens and Michael generalized the theory of Banach algebras by introducing the concept of a locally multiplicatively-convex topological algebra (abbreviated "Imc" algebra). On the other hand, Arens gave a procedure for defining on the bidual (the second topological conjugate space) of a Banach algebra a multiplication which makes the bidual also into a Banach algebra. We show that one can put an Arens multiplication onto the bidual of an Imc algebra, and we study the algebraic and topological properties of the bidual when it is endowed with such a multiplication.

In $\S 2$ we summarize briefly the basic definitions and elementary results needed in the paper. Section 3 contains the main construction of the paper, the construction in the bidual of an lme algebra of a multiplication which is an extension of the multiplication on the original algebra. For the sake of generality we actually make the construction on the bidual of any topological algebra whose multiplication is hypo-continuous. Under this multiplication the bidual is always a topological algebra. If the bidual is even an lmc algebra we call the given algebra a bi-lme algebra. We show that a necessary and sufficient condition that an lmc algebra be a bi-lmc algebra is that the given algebra be lme under the strong topology. Consequently any quasi-barreled algebra-hence any $F$ algebra-is a bi-lme algebra. We prove that the property of being a bi-lmc algebra is preserved in passing to certain related algebras. We end the section by exhibiting two lme algebras which are not quasi-barreled but which are bi-lmc algebras.

A complete lme algebra has associated sequences of Banach algebras [18, Theorem 5.1]. For a given complete lmc algebra, assume that its bidual is a complete lme algebra; then a natural question to ask is how the associated sequences for the lmc algebra and for its bidual are related. We devote $\S 4$ to this matter. We provide a necessary and sufficient condition for a sequence in the bidual of an $F$ algebra

Received November 7, 1963, and in revised form June 16, 1964. Part of this material is drawn from a dissertation written at Yale University. The research was partially supported by the Air Force Office of Scientific Research Grant $62-20$. 
to contain precisely the biduals of the members of a sequence for the base algebra. Finally, we form a new type of nonnormable $F$ algebra, which has the topological properties of $L_{1}$ spaces.

A question which has merited attention is whether or not the bidual of a given commutative Banach algebra is again commutative. Arens [3] proved that the bidual of a commutative $B^{*}$ algebra is also a commutative $B^{*}$ algebra, and Kamowitz [15] proved that any multiplication which makes a $B^{*}$ algebra into a commutative Banach algebra renders the bidual commutative. On the other hand, Civin and Yood [8] showed that the bidual of a commutative group algebra is never commutative unless the group is finite. Nevertheless, we have as yet no criterion on an lme algebra which determines whether or not the bidual is commutative, even if the given lme algebra is normed. Any Imc algebra whose bidual is commutative we call a bicommutative algebra. The main theorem in $\S 5$ is that if each member of an associated sequence for a complete lmc algebra is bicommutative, then the lme algebra is bicommutative. The theorem remains true regardless of whether or not the lme algebra is a bi-lme algebra. We generalize a theorem of Civin and Yood [8] and show that subalgebras of bicommutative algebras are also bicommutative. We also show that under some mild changes in multiplication, the property of being or of not being bicommutative is retained.

In $\S 6$ we present two examples. Our first is a continuous function algebra whose bidual is commutative and has nonzero radical. Our second, the one described in $\S 4$, is shown to be a nonbicommutative $F$ algebra whose bidual has also a nonzero radical.

2. Preliminary discussion. Throughout this paper, $E=(E, t)$ will denote a locally convex, Hausdorff, linear topological space over the complex numbers, where $E$ is the vector space and where $t$ is the locally convex, Hausdorff topology. As in [18], a basis for $E$ consists of a collection of closed, convex, equilibrated subsets of $E$ whose scalar multiples form a basis for the neighborhoods of the origin 0 in $E$. By a bounded system for $E$ we mean a collection $\mathscr{B}$ of closed, convex, equilibrated, bounded sets in $E$, such that for any bounded set $B$ in $E$ there is a member of $\mathscr{B}$ which absorbs $B$. Let $E$ and $F$ be two linear topological spaces, and let $A \subseteq E$ and $B \subseteq F$. We say that $A$ and $B$ are linearly isomorphic if there is a one-to-one map from $A$ onto $B$ and if the map is linear. $A$ and $B$ are topologically isomorphic if they are homeomorphic. If $E$ and $F$ are in addition algebras, then $A$ and $B$ are algebraically isomorphic if they are linearly isomorphic by a map which at the same time preserves multiplication. Finally, if $A$ and $B$ are linearly and topologically (and algebraically if possible) isomorphic, we say that $A$ and $B$ are equivalent, or $A \cong B$. 
Let $E$ be an algebra. A subset $A$ of $E$ is idempotent if $A A \cong A$. A topological algebra is a linear topological space which is an (associative) algebra and which has the property that ring multiplication is separately continuous. Then we define $E$ to be a locally multiplicatively-convex topological (hereafter abbreviated $l m c$ ) algebra if $E$ is a topological algebra and if there exists a basis for $E$ consisting of convex sets which are idempotent. Normed algebras and locally convex linear topological spaces with zero multiplication are lmc algebras. An $F$ algebra (resp. $F$ space) is a metrizable and complete lmc algebra (resp. locally convex space). If $\left(U_{n}\right)_{n=1}^{\infty}$ is a basis, we assume that for all $n, U_{n} \supset U_{n+1}$, and $U_{n}$ is not absorbed by $U_{n+1}$. An example of an $F$ algebra is the space of continuous, complex-valued functions on $[0,1)$ under pointwise multiplication and with the compact-open topology. An $L B$ space is a strict inductive limit of a countably infinite collection of Banach spaces. The collection $\mathscr{E}$ (or any collection $\mathscr{F}$ in one-to-one correspondence with $\mathscr{E}$ such that each member of $\mathscr{F}$ is equivalent to its corresponding member in $\mathscr{E}$ ) of Banach spaces in the inductive limit space $E$ is called a defining sequence for $E$. Let $E$ be a complete lmc algebra, with basis $\left(V_{\lambda}\right)_{\lambda \in \Lambda}$. For each $\lambda \in \Lambda$, let $P_{\lambda}$ be the Minkowski functional for $V_{\lambda}$, and define $\widetilde{E}_{\lambda}=\left(E / p_{\lambda}^{-1}(0)\right.$, $\left.p_{\lambda}^{\prime}\right)$, where $p_{\lambda}^{\prime}$ is the norm on $E / p_{\lambda}^{-1}(0)$ corresponding to the functional $P_{\lambda}$ on $E$. Let $E_{\lambda}$ be the completion of $\widetilde{E}_{\lambda}$ with respect to $p_{\lambda}^{\prime}$. Then $E_{\lambda}$ is a Banach algebra, and $\left(E_{\lambda}\right)_{\lambda \in 1}$ is an associated sequence (of Banach algebras) for $E$.

By the dual of $E$ we mean the space of all continuous, complexvalued linear forms on $E$. We denote the dual of $E$ by $E^{*}$, and the members of $E^{*}$ by $x^{*}, y^{*}$, etc. The dual (also called $t_{b}^{*}$ or $B\left(E^{*}, E\right)$ ) topology on $E^{*}$ is the topology of uniform convergence on the bounded sets of $E$. Ordinarily the dual $E^{*}$ has the dual topology. The weak-(E) (or $t_{s}^{*}$ ) topology on $E^{*}$ is the topology of uniform convergence on finite point sets of $E$. On $E$ there is the strong (resp. weak- $\left(E^{*}\right)$ ) topology, denoted by $t_{b}$ (resp. $t_{s}$ ), which is the topology of uniform convergence on any bounded system (resp. finite point sets) of $E^{*}$. Always $t_{b}$ is stronger than $t$; if $t_{b}=t$ then $E$ is quasi-barreled. Every metrizable space is quasi-barreled. The largest locally convex topology on $E$ which yields $E^{*}$ as dual is called the Mackey topology, written $\tau(E$, $\left.E^{*}\right)$. Again, metrizable spaces have the Mackey topology. Let $E$ be a complete Imc algebra with $\left(V_{\lambda}\right)_{\lambda \in \Lambda}$ as basis and $\left(E_{\lambda}\right)_{\lambda \in \Lambda}$ an associated sequence for $E$. For each $\lambda \in \Lambda, E_{\lambda}^{*}=\left(E_{\lambda}\right)^{*}$ is linearly isomorphic to a subspace of $E^{*}$ by the equation $x_{\lambda}^{*}\left(x+p_{\lambda}^{-1}(0)\right)=x^{*}(x)$ for all $x \in E$ [11, p. 98]. Since the topology of $E$ is stronger than the topology on the semi-normed space from which $E_{\lambda}$ arises, it is apparent that the $B\left(E_{\lambda}^{*}, E_{\lambda}\right)$ topology on $E_{\lambda}^{*}$ is stronger than the restriction to $E_{\lambda}^{*}$ of 
the $B\left(E^{*}, E\right)$ topology on $E^{*}$. For a set $A$ in $E$, the polar in $E^{*}$ of $A$ is defined as $A^{\delta}=\left\{x^{*} \in E^{*}:\left|x^{*}(x)\right| \leqq 1\right.$ for all $\left.x \in A\right\}$. Likewise, for a set $A^{*}$ in $E^{*}$, the polar in $E$ of $A^{*}$ is defined as $A^{* 9}=$ $\left\{x \in E:\left|x^{*}(x)\right| \leqq 1\right.$ for all $\left.x^{*} \in A^{*}\right\}$. It is well-known that if $A \subseteq E$, then $A^{\text {d }}$ i is the weak-( $\left.E^{*}\right)$ closed, convex, equilibrated hull of $A$. It follows immediately from our definitions that the dual and weak- $(E)$ topologies on $E^{*}$ have for bases the polars of a bounded system and the finite point sets in $E$, respectively.

The bidual of a locally convex linear topological space $E$ is the dual of the dual $E^{*}$ (with dual topology), and is denoted $E^{* *}$, with elements written $x^{* *}, y^{* *}$, etc. The bidual topology is the topology of uniform convergence on bounded sets of $E^{*}$. The bidual normally has the bidual topology. If $A$ is a set in $E$, then the bipolar of $A$ in $E^{* *}$ is $A^{\delta \delta}=\left(A^{\circ}\right)^{b}$. Now if $U$ is a neighborhood of 0 in $E$, then $U^{\delta}$ is a bounded set in $E^{*}$, so that $U^{\delta} \delta$ is a neighborhood of 0 in $E^{* * *}$. In general, however, if $\left(U_{\lambda}\right)_{\lambda \in_{\Lambda}}$ is a basis for $E$, then $\left(U_{\lambda}^{\delta}\right)_{\lambda \in \Lambda}$ is not a basis for $E^{* *}$. As a matter of fact, it is if and only if $E$ is quasi-barreled. A second topology on $E^{* *}$ is the weak- $\left(E^{*}\right)$ topology of uniform convergence on finite point sets of $E^{*}$. Let us define the function $\hat{x}$ on $E^{*}$ corresponding to an arbitrary element $x$ in $E$ by the relation $\hat{x}\left(x^{*}\right)=x^{*}(x)$, for all $x^{*} \in E^{*}$. Clearly $\hat{x}$ is linear, and is continuous for the weak- $(E)$ topology on $E^{*}$, and hence for the stronger dual topology on $E^{*}$. Consequently $\hat{x}$ is in $E^{* *}$. We define $\hat{E}$ to be the linear, one-to-one embedding of $E$ into $E^{* *}$, where $x$ in $E$ is mapped onto $\hat{x}$ in $E^{* *}$. Then $\hat{E}$ is weak- $\left(E^{*}\right)$ dense in $E^{* *}[17, \mathrm{p}$. 300]. This result is of prime importance when we show that our multiplication on the bidual of an lme algebra is associative.

3. The bidual of an LMC algebra as an algebra. In this section we first construct a multiplication on the bidual of certain topological algebras. We assume that the multiplication is always hypo-continuous, i.e., for each neighborhood $U$ of 0 and for each bounded set $B$ there is a second neighborhood $V$ of 0 such that $V \circ B \cong U$. Although this requirement is sufficient it is not necessary. However, the author feels it is the weakest manageable condition which insures a multiplication on the bidual of a topological algebra. From here on through Theorem 3.8 , let $(E, \circ)$ be an arbitrary but fixed algebra with hypo-continuous multiplication $\circ .^{1}$

Notation. Though "॰" will appear in several contexts as a bilinear function, we will always know on what spaces o operates if we look at the elements to the left and to the right of $\circ$.

1 The author thanks the referee for his suggestions for this section. 
Lemma 3.1. Let an operation “o”, defined on $\left(E^{*}, E\right)$ be given by

$$
\left(x^{*} \circ x\right) y=x^{*}(x \circ y), \quad y \in E,
$$

where $x^{*} \in E^{*}$ and $x \in E$. Then for any $x^{*} \in E^{*}$ the linear transformation $x \rightarrow x^{* \circ} x$ is continuous from $E$ into $E^{*}$ for the dual topology on $E^{*}$.

Proof. Left multiplication is continuous in $E$, so $x^{* \circ} \circ \in E^{*}$ for any $x \in E$. The linearity of the map $x \rightarrow x^{*} \circ x$ is obvious. To show that the map is continuous, let $B$ be a bounded subset of $E$. Since $x^{*}$ is continuous, there exists a neighborhood $U$ of 0 in $E$ such that $U \subseteq\left\{x^{*}\right\}^{\text {? }}$. But multiplication in $E$ is hypo-continuous, so consequently there is a neighborhood $V$ of 0 in $E$ such that $V \circ B \cong U$. Then for any $x \in V,\left|\left(x^{*} \circ x\right) B\right| \leqq 1$, whence $x \rightarrow x^{*} \circ x$ is continuous.

Lemma 3.2. Let an operation “o" defined on (E**, $\left.E^{*}\right)$ be given by

$$
\left(y^{* * \circ} \circ x^{*}\right) x=y^{* *}\left(x^{*} \circ x\right), \quad x \in E,
$$

where $y^{* *} \in E^{* *}$ and $x^{*} \in E^{*}$. Then for any $y^{* *} \in E^{* * *}$, the linear transformation $x^{*} \rightarrow y^{* *} \circ x^{*}$ is continuous from $E^{*}$ into $E^{*}$ for the dual topology on $E^{*}$.

Proof. We note first that $y^{* *} \circ x^{*}$ is the composite of $x \rightarrow x^{*} \circ x$ and $x^{*} \circ x \rightarrow y^{* *}\left(x^{*} \circ x\right)$, each of which is continuous with respect to the dual topology of $E^{*}$. Hence $y^{* *} \circ x^{*} \in E^{*}$ for any $x^{*} \in E^{*}$. The map $x^{*} \rightarrow y^{* * \circ} \circ x^{*}$ is linear, and to show it is continuous, let $B$ be a bounded subset of $E$. Since $y^{* *} \in E^{* *}, C^{b} \cong\left\{y^{* *}\right\}^{9}$ for some bounded $C$ in $E$. Then $x^{*} \in(B \circ C)^{\circ}$, implies $\left(x^{*} \circ B\right) \subseteq C^{\circ}$, whence $y^{* *} \circ x^{*} \in B^{\delta}$.

Lemma 3.3. For any $x^{*} \in E^{*}$, the map $y^{* *} \rightarrow y^{* *} \circ x^{*}$ from $E^{* *}$ into $E^{*}$ is continuous for the bidual and dual topologies on $E^{* *}$ and $E^{*}$ respectively.

Proof. Since $x^{*} \circ x \in E^{*}$ for any $x \in E$, the map is continuous for the weak- $\left(E^{*}\right)$ and weak- $(E)$ topologies $E^{* *}$ and $E^{*}$ respectively. Then by Proposition 6 in [7], the map is continuous for the bidual and dual topologies on $E^{* *}$ and $E^{*}$ respectively.

LEMmA 3.4. Let an operation “o" defined on $\left(E^{* *}, E^{* *}\right)$ be given by

$$
\left(x^{* * \circ} \circ y^{* *}\right) x^{*}=x^{* *}\left(y^{* *} \circ x^{*}\right), \quad x^{*} \in E^{*},
$$


where $x^{* *}, y^{* *} \in E^{* *}$. Then for any $y^{* *} \in E^{* *}$, the linear transformation $x^{* *} \rightarrow x^{* *} \circ y^{* *}$ is continuous for the weak- $\left(E^{*}\right)$ and bidual topologies on $E^{* *}$.

Proof. Again the linearity is obvious. The maps $x^{*} \rightarrow y^{* *} \circ x^{*}$

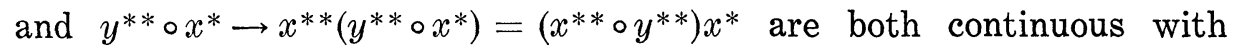
respect to the dual topology on $E^{*}$, so their composite is also continuous, whence $x^{* *} \circ y^{* *} \in E^{* *}$. If $x^{*} \in E^{*}$, then $y^{* *} \circ x^{*} \in E^{*}$, so that $x^{* *} \rightarrow x^{* *} \circ y^{* *}$ is continuous for the weak- $\left(E^{*}\right)$ topology on $E^{* *}$. Then Proposition 6 in [7] implies that the map is continuous for the bidual topology on $E^{* *}$.

THEOREM 3.5. With the multiplication defined on $E^{* *}$ by Lemma $3.4, E^{* *}$ is an algebra, and the $\operatorname{map} x \rightarrow \widehat{x}$ is an algebraic isomorphism of $\hat{E}$ onto $E$ in $E^{* *}$.

Proof. With the definition of multiplication given in Lemma 3.4, $E^{* *}$ is an algebra, by virtue of the linearity of the map in Lemma 3.3 , and by definition of sums of functions. Next, if $x, y \in E$ and $x^{*} \in E^{*}$, then $(\hat{x} \circ \hat{y}) x^{*}=x^{*}(x \circ y)=x(\widehat{x \circ y}) x^{*}$, by the various definitions of $\circ$. Thus the linear isomorphism embedding $x \rightarrow \widehat{x}$ of $E$ onto $\hat{E}$ in $E^{* *}$ is an algebraic isomorphism.

Lemma 3.6. For any $x \in E$, the map $y^{* *} \rightarrow \widehat{x} \circ y^{* *}$ of $E^{* *}$ into $E^{* *}$ is continuous for the weak-( $\left.E^{*}\right)$ and bidual topologies on $E^{* *}$.

Proof. Note that for any $y^{* *} \in E^{* *}, x^{*} \in E^{*}$, and $x \in E$, we have $\left(\hat{x} \circ y^{* *}\right) x^{*}=y^{* *}\left(x^{*} \circ x\right)$, so that the map $y^{* *} \rightarrow \hat{x} \circ y^{* *}$ is continuous for the weak-( $\left.E^{*}\right)$ topology on $E^{* *}$, and then by Proposition 6 of [7] it is continuous for the bidual topology on $E^{* *}$.

Lemma 3.7. For any $x^{* *} \in E^{* *}$, the map $y^{* *} \rightarrow x^{* *} \circ y^{* *}$ of $E^{* *}$ into $E^{* *}$ is continuous for the bidual topology on $E^{* *}$.

Proof. Let $C^{*}$ be a bounded subset of $E^{*}$, and let $B$ be a bounded subset of $E$. If $A$ is also bounded in $E$, then since multiplication in $E$ is hypo-continuous, $B \circ A$ is bounded, so there is a $\lambda>0$ such that $C^{*} \subseteq \lambda(B \circ A)^{\dagger}$. Since $\left(C^{*} \circ B\right) A=C^{*}(B \circ A)$, we have $C^{*} \circ B \subseteq \lambda A^{\delta}$, whence $C^{*} \circ B$ is bounded in $E^{*}$. Because $x^{* *} \in E^{* *}$, there is such a bounded set $B$ in $E$ for which $B^{\delta} \subseteq\left\{x^{* *}\right\}^{9}$. Now let $y^{* *} \in\left(C^{*} \circ B\right)^{b}$.

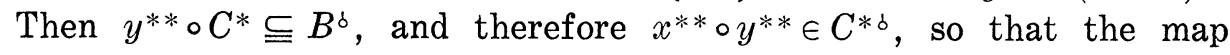
$y^{* *} \rightarrow x^{* *} \circ y^{* *}$ is continuous. 
THEOREM 3.8. The multiplication of $E^{* *}$ is separately continuous.

Proof. Follows from Lemmas 3.4 and 3.7 .

THEOREM 3.9. If $E$ is an associative algebra, the $E^{* *}$ is also an associative algebra.

Proof. Let $x, y \in E$ and $z^{* *} \in E^{* *}$. The functions $z^{* *} \rightarrow \widehat{x} \circ\left(\hat{y} \circ z^{* *}\right)$ and $z^{* *} \rightarrow(\hat{x} \circ \hat{y}) \circ z^{* *}$ are continuous for the weak- $\left(E^{*}\right)$ topology, by repeated application of Lemma 3.6. By the algebraic isomorphism, $E$ onto $\hat{E}$, they coincide on $\hat{E}$. Thus the density of $\hat{E}$ in $E^{* *}$ with respect to the weak $-\left(E^{*}\right)$ topology yields $\hat{x} \circ\left(\hat{y} \circ z^{* *}\right)=(\hat{x} \circ \hat{y}) \circ z^{* *}$ for all $z^{* *} \in E^{* *}$. Next, let $\hat{x} \in E$ and $z^{* *} \in E^{* *}$. The functions $y^{* *} \rightarrow$ $\hat{x} \circ\left(y^{* *} \circ z^{* *}\right)$ and $x^{* *} \rightarrow\left(\hat{x} \circ y^{* *}\right) \circ z^{* *}$ are continuous on $E^{* *}$ for the weak- $\left(E^{*}\right)$ topology, by recourse to Lemmas 3.4 and 3.6 . By the preceding sentence, these maps coincide on $\hat{E}$, so once again they coincide on $E^{* *}$, which means that $\hat{x} \circ\left(y^{* *} \circ z^{* *}\right)=\left(\widehat{x} \circ y^{* *}\right) \circ z^{* *}$ for all $y^{* *} \in E^{* *}$. Finally, let $y^{* *}, z^{* *} \in E^{* *}$. The functions $x^{* *} \rightarrow$ $x^{* *} \circ\left(y^{* *} \circ z^{* *}\right)$ and $x^{* *} \rightarrow\left(x^{* *} \circ y^{* *}\right) \circ z^{* *}$ are continuous on $E^{* *}$ for the weak- $\left(E^{*}\right)$ topology by Lemma 3.4 , and they coincide on $\hat{E}$ by the preceding statement. Hence they coincide on all of $E^{* *}$, which just means that $E^{* *}$ is associative.

THEOREM 3.10. $E^{* *}$ has jointly continuous (locally m-convex) multiplication if and only if E, equipped with strong topology, has jointly continuous (locally m-convex) multiplication.

Proof. Let $A, B, C \subseteq E$ such that $A \circ B \subseteq C$, and let all closures be with respect to the weak- $\left(E^{*}\right)$ topology. For each $x \in A$, we have $\hat{x} \circ \overline{\hat{B}} \cong \overline{\hat{C}}$ by Lemma 3.6. Thus for each $y^{* *} \in \overline{\hat{B}}$, we have $\overline{\hat{A}} \circ y^{* *} \cong \overline{\hat{C}}$, by Lemma 3.4. Thus $\overline{\hat{A}} \circ \overline{\hat{B}} \subseteq \overline{\hat{C}}$. If $E$ has the strong topology, then the map $x \rightarrow \hat{x}$ is a topological isomorphism between $E$ and $\hat{E}$. Furthermore, the closures for the weak- $\left(E^{*}\right)$ topology of the members of a fundamental system of neighborhoods of 0 in $E$ is a fundamental system of neighborhoods of 0 in $E^{* *}$. Thus continuity of multiplication on $E^{* *}$ is inherited from continuity of multiplication on $E$ when it is endowed with the strong topology.

REMARK 3.11. Let $z \in E$. Then the maps $x^{* *} \rightarrow x^{* * \circ} \widehat{z}$ and $y^{* *} \rightarrow \hat{z} \circ y^{* *}$ are continuous on $E^{* *}$ for the weak- $\left(E^{*}\right)$ topology by Lemmas 3.4 and 3.6. Since the maps coincide on $\hat{E}$, the weak-( $\left.E^{*}\right)$ density of $\hat{E}$ in $E^{* *}$ proves that they coincide on $E^{* *}$. This means 
that the center of $E$ is embedded in the center of $E^{* *}$. Furthermore, if we assume that $E$ is commutative, then $E^{* *}$ is commutative if and only if the map $y^{* *} \rightarrow x^{* *} \circ y^{* *}$ is continuous for the weak- $\left(E^{*}\right)$ topology (i.e., if left multiplication in $E^{* *}$ is weak-( $\left.E^{*}\right)$ continuous). This follows from the fact that the maps $y^{* *} \rightarrow x^{* *} \circ y^{* *}$ and $y^{* *} \rightarrow y^{* * \circ} \circ x^{* *}$ agree on $\hat{E}$, and the latter is weak- $\left(E^{*}\right)$ continuous. We remark also that the second adjoint of a continuous multiplicative linear form on $E$ is a continuous multiplicative linear form on $E^{* *}$, by an argument which parrots Lemma 3.6 in [8].

ExAmPles 3.12. We give an example of a topological algebra for which the Arens multiplication on its bidual cannot be constructed. Let $E$ be the algebra of all continuous, complex-valued functions defined and continuous on the interval $[0,1]$, under pointwise multiplication and endowed with the restricted $L_{1}[0,1]$ topology. Then on $E$ the multiplication is separately continuous but not hypo-continuous. If $x^{*} \in E^{*}$ and $x \in E$, then $E^{*}$ corresponds to $L_{\infty}[0,1]$ by the map $x^{*} \rightarrow h_{x^{*}}$ where $x^{*}(x)=\int_{0}^{1} h_{x^{*}}(t) d t$ (see [12, Theorem IV. 8.5]). Then for almost all $t$ in $[0,1], h_{x^{*} \circ x}(t)=h_{x^{*}}(t) x(t)$, so that "o" is defined on $\left(E^{*}, E\right)$. Next, if $x^{* *} \in E^{* *}$ and $x^{*} \in E^{*}$, then $E^{* *}$ corresponds to the Banach space of all finitely additive measures on $[0,1]$ by the map $x^{* *} \rightarrow m_{x^{* *}}$ where $x^{* *}\left(x^{*}\right)=\int_{0}^{1} h_{x^{*}}(t) m_{x^{* *}}(d t) \quad$ (see [12, Theorem IV. 8.16]). Then $\left(x^{* *} \circ y^{*}\right) x=\int_{0}^{1} h_{y^{*}}(t) x(t) m_{x^{* *}}(d t)$ for all $x^{* *} \in E^{* *}, y^{*} \in E^{*}$, and $x \in E$. Now for all $n$ define $x_{n}$ by

$$
x_{n}(t)=\left\{\begin{array}{ll}
-2 n^{2} t+2 n, & 0 \leqq t \leqq 1 / n \\
0, & 1 / n<t \leqq 1
\end{array}\right\} .
$$

Note that $x_{n} \in E$ and $\left\|x_{n}\right\|=1$, for all $n$. Let $x^{* *} \in E^{* *}$ be a weak- $\left(E^{*}\right)$ cluster point of $\left(x_{n}\right)_{n=1}^{\infty}$. Let $y^{*} \in E^{*}$ correspond to $h_{y^{*}}$ where $h_{y^{*}}(t)=1$ for all $t$ in $[0,1]$. Then $\lim _{n \rightarrow \infty}\left(x^{* *} \circ y^{*}\right) x_{n}=\infty$, so that $x^{* * \circ} \circ y^{*} \notin E^{* *}$, and the second phase of "o" cannot be defined.

On the other hand, we display an example of a topological algebra with multiplication which is hypo-continuous but not jointly continuous. It is the algebra of continuous, complex-valued functions on $[0,1]$, under pointwise multiplication, and with the weak topology resulting from the sup norm topology (see [22]).

Finally we exhibit a topological algebra whose multiplication is jointly continuous but not locally $m$-convex. We let $E$ be the algebra of continuous, complex-valued functions on $[0,1]$, under pointwise multiplication. This time we take for a basis the collection of sets 


$$
\begin{gathered}
V_{\left(m_{n}\right)}=\left\{x \in E:|x(t)| \leqq m_{n}, \text { all } t \text { in }[1-1 / n, \quad 1-1 /(n+1)],\right. \\
n=1,2, \cdots\},
\end{gathered}
$$

where $0<m_{1}<m_{2}<\cdots$ and $\lim _{n \rightarrow \infty} m_{n}=\infty$.

From now on we restrict our attention to lme algebras. We have next

DeFinItion 3.13. A bi-lme algebra is an lme algebra whose bidual is also an lme algebra.

We conjecture that not every lme algebra is a bi-lme algebra. On the other hand, certainly any lme algebra with zero multiplication is a bi-lme algebra, since all the various bilinear forms inducing the multiplication on the bidual will be identically zero. In addition, any quasi-barreled lmc algebra (thus any $F$ algebra or reflexive lmc algebra) is a bi-lmc algebra, since the strong topology on a quasi-barreled algebra is the same as the original topology.

If $E$ is an lme algebra, then we define $E^{+}$to be the lme algebra which is the cartesian product of $E$ and the complex numbers, with the following multiplication on $E^{+}$: if $x, y \in E$, and if $a$ and $b$ are complex numbers, then $(x, a) \circ(y, b)=(x \circ y+a y+b x, a b)$. Then $E^{+}$ is an lme algebra by Proposition 2.4 of [18].

Theorem 3.14. An lme algebra $E$ is bi-lme if and only if $E^{+}$ is bi-lmc.

Proof. By Michael's Proposition 2.4 it is enough for us to show that $E^{+* *}=E^{* *+}$. Let members of $E^{+*}$ have the form $x^{*}+c$, where $x^{*} \in E^{*}$ and $c$ is a complex number. Now to the computation of multiplications on $E^{+* *}$ and on $E^{* *+}$. Let $\left(x^{*}+c\right) \in E^{+*}$ and $(x, d) \in E^{+}$. Then for $(y, e) \in E^{+}$we have

$$
\begin{aligned}
{\left[\left(x^{*}+c\right) \circ(x, d)\right](y, e) } & =\left\{\left(x^{*}+c\right)\right\}[(x \circ y+d y+e x, d e)] \\
& =x^{*}(x \circ y+d y+e x)+c d e \\
& =\left(x^{*} \circ x\right) y+d x^{*}(y)+e x^{*}(x)+c d e \\
& =\left\{\left[\left(x^{*} \circ x\right)+d x^{*}\right]+\left[x^{*}(x)+c d\right]\right\}(y, e),
\end{aligned}
$$

so that $\left(x^{*}+c\right) \circ(x, d)=\left[\left(x^{*} \circ x\right)+d x^{*}\right]+\left[x^{*}(x)+c d\right]$. In the same vein, if $\left(y^{* *}, b\right) \in E^{+* *}$ and $\left(x^{*}+c\right) \in E^{+*}$, then $\left(y^{* *}, b\right) \circ\left(x^{*}+c\right)=$ $\left[\left(y^{* *} \circ x^{*}\right)+b x^{*}\right]+\left[y^{* *}\left(x^{*}\right)+b c\right]$. Finally, for $\left(x^{* *}, a\right)$ and $\left(y^{* *}, b\right)$ in $E^{+* *}$, a similar computation yields

$$
\left(x^{* *}, a\right) \circ\left(y^{* *}, b\right)=\left(\left(x^{* *} \circ y^{* *}\right)+b x^{* *}+a y^{* *}, a b\right) .
$$

But since $E^{+* *}$ and $E^{* *+}$ are anyway linearly and topologically isomorphic, this shows they are equivalent. 
Theorem 3.15. The Cartesian product $\prod_{\lambda \in A} E_{\lambda}$, with product topology, of lmc algebras $E_{\lambda}, \lambda \in \Lambda$, is a bi-lmc algebra if and only if for each $\lambda \in \Lambda, E_{\lambda}$ is a bi-lmc algebra.

Proof. The dual of a cartesian product (respectively direct sum) of locally convex spaces is the direct sum (respectively cartesian product) of the locally convex spaces, as shown in [16, V.18.10]. Consequently the bidual of a cartesian product of locally convex spaces is the cartesian product of the biduals of these same locally convex spaces. By Proposition 2.4 of [18], subalgebras and products of lmc algebras are Ime algebras, so our assertion follows.

We remark in passing that to show that every lmc algebra is a bi-lmc algebra, it would suffice to prove that arbitrary subalgebras of of bi-lmc algebras are bi-lme algebras, because by Proposition 2.7 of [18] a topological algebra is lme if and only if it is topologically and algebraically isomorphic to a subalgebra of a cartesian product of normed algebras. From our Theorem 3.9 any cartesian product of normed algebras is a bi-lmc algebra, since normed algebras are evidently bi-lme algebras. We mention too that the problem of telling when lmc algebras remain lme when endowed with the strong topology is related to questions posed by Warner in [21] and [22]. In [22] he finds necessary and sufficient conditions for $\left(E, t_{s}\right)$ to be lmc. In [21] he leaves as an open question one which is equivalent to the following: is an lme algebra still lme if it is endowed with the Mackey topology?

We present here two examples of non-trivial, non-quasi-barreled lme algebras which are bi-lme algebras. We use Theorem 3.6 to show this fact.

EXAMPLE 3.16. Let $[0,1]$ have the usual topology, and let $E$ be the algebra $C[0,1]$ of continuous, complex-valued functions on $[0,1]$ under pointwise multiplication, with the topology of uniform convergence on subsets of $[0,1)$ which are simultaneously countable and compact. By a moment's reflection we see that $E$ is an lme algebra. First we characterize the bounded sets in $E$. Let $\alpha$ be a nondecreasing function from the positive integers into the positive integers, and let $A$ be the collection of such maps. Let $\alpha \in A$ and let

$B_{\alpha}=\{x \in E:|x(t)| \leqq \alpha(n)$ for all $t$ in $[0,1-1 / n]$ and for all $n\}$. It is apparent that each such $B_{\alpha}$ is bounded in $E$, since any countable and compact subset $S$ of $[0,1)$ is contained in $[0,1-1 / n]$ for some $n$, and $B_{\alpha}$ is uniformly bounded on $S$. On the other hand, let $D \leqq E$ and assume that $D$ is not bounded uniformly on $[0,1-1 / n]$, for some $n$. Then there is a sequence $\left(t_{m}\right)_{m=1}^{\infty}$ in $[0,1-1 / n]$ and a sequence $\left(x_{m}\right)_{m=1}^{\infty}$ in $D$ such that for each $m,\left|x_{m}\left(t_{m}\right)\right|>m$. Now $\left(t_{m}\right)_{m=1}^{\infty}$ has a subsequence $\left(t_{m_{k}}\right)_{k=1}^{\infty}$ which converges to a unique limit $t_{0}$ in $[0,1-1 / n]$. 
If we let $S=\left[\left(t_{m_{k}}\right)_{k=1}^{\infty}\right] \cup\left\{t_{0}\right\}$, then $S$ is countable and compact, and $D$ is not bounded uniformly on $S$, and hence $D$ is not bounded in $E$. Thus any bounded set in $E$ is contained in $B_{\alpha}$, for some $\alpha \in A$. This means that $\left(B_{\alpha}\right)_{\alpha \in A}$ is a bounded system for $E$. Let $F$ be the algebra $C[0,1)$ under pointwise multiplication, with compact-open topology. Then $F$ is an $F$ algebra with basis $\left(V_{n}\right)_{n=1}^{\infty}$, where $V_{n}=\{x \in F$ : $|x(t)| \leqq 1, t \in[0,1-1 / n]\}$. It is easily shown that $F$ has the same bounded sets as $E$. Thus $\left(B_{\alpha}\right)_{\alpha \in A}$ serves as a bounded system for $F$. Using this fact, as well as the fact that $F$ has a stronger topology then $E$, it is apparent that $E^{*}$ is (topologically as linearly) a subspace of $F^{*}$. Now $\left(V_{n}^{b}\right)_{n=1}^{\infty}$ may be chosen as a bounded system for $F^{*}$, because $F$ is quasi-barreled. Therefore $\left(V_{n}^{\delta} \cap E^{*}\right)_{n=1}^{\infty}$ constitutes a bounded system for $E^{*}$, and thus $\left\{\left(V_{n}^{b} \cap E^{*}\right)^{9}\right\}_{n=1}^{\infty}$ is a basis for $\left(E, t_{b}\right)$. We now show that for each $n$, $\left(V_{n}^{b} \cap E^{*}\right)^{9}=V_{n}$. Certainly

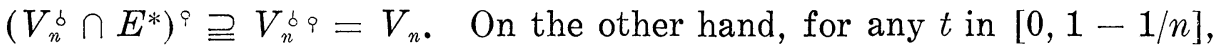
let $m_{t}$ be the point mass at $t$, with $m_{t}\{t\}=1$. Then $m_{t}$ is in $V_{n}^{b} \cap E^{*}$, so that if $x \in\left(V_{n}^{\delta} \cap E^{*}\right)^{9}$, then $|x(t)|=\left|m_{t}(x)\right| \leqq 1$, whence $x \in V_{n}$. Thus $\left(V_{n}^{\dot{\delta}} \cap E^{*}\right)^{?}=V_{n}$, and the latter is idempotent. By recourse to Theorem 3.6, we have $E$ a bi-lme algebra.

ExAmple 3.17. Let $A$ be an uncountable indexing set, and let $\Pi_{\lambda \in \Lambda} E_{\lambda}$ be the cartesian product (without topology) of the $E_{\lambda}$, where each $E_{\lambda}$ is a copy of the complex numbers. Let a basis for $\Pi_{\lambda \in \Lambda} E_{\lambda}$ consist of $\prod_{\lambda \in A} V_{\lambda}$, where for each $\lambda \in \Lambda, V_{\lambda}$ is any neighborhood of 0 in $E_{\lambda}$ which is contained in the unit sphere of $E_{\lambda}$ with its usual norm topology. Now let $E$ be the direct sum of $\Pi_{\lambda \in A} E_{\lambda}$, i.e., all $x=\left(x_{\lambda}\right)_{\lambda \in A}$ in $\prod_{\lambda \in \Lambda} E_{\lambda}$ such that $x_{\lambda}=0$ except for a finite set of indices. Let $E$ have the topology induced from $\prod_{\lambda \in \Lambda} E_{\lambda}$, and multiplication consisting of coordinate-wise multiplication. Then $E$ is a Hausdorff locally convex space [6, p. 22]. Since each $V_{\lambda}$ is idempotent, it is clear that $E$ is lmc. We will show that $E$ is a bi-lme algebra, although it is not quasi-barreled. We observe that bounded sets in $E$ are of the form $\left(\prod_{\lambda \in \Lambda} B\right) \cap E$, where for each $\lambda \in \Lambda, B_{\lambda}$ is bounded in $E_{\lambda}$, and $B_{\lambda}=(0)$ except for a finite set of indices [7, p. 12]. That $E$ is not quasibarreled follows from the fact that the set $R=\left\{x \in E: \sum_{\lambda \epsilon_{\Lambda}}\left|x_{\lambda}\right| \leqq 1\right\}$ is a barrel which absorbs all bounded sets of $E$; but $R$ is not a neighborhood of 0 in $E\left[7\right.$, p. 3]. For a set $D_{\lambda} \subseteq E_{\lambda} \cong E_{\lambda}^{*}$, let $\left|D_{\lambda}\right|=$ $\sup \left\{\left|x_{\lambda}\right|: x_{\lambda} \in D_{\lambda}\right\}$ and let $D_{\lambda}^{\prime}=\left\{x:|x| \leqq 1 /\left|D_{\lambda}\right|\right\}$. Let $B=\Pi_{\lambda \in_{\Lambda}} B_{\lambda}$ be bounded in $E$, so that $B_{\lambda}=(0)$ except for a finite subset $\lambda_{1}, \cdots, \lambda_{n}$ of $\Lambda$. Then $B \subseteq C=\prod_{\lambda \in A} C_{\lambda}$, where

$$
C_{\lambda_{i}}=\left\{x_{\lambda_{i}} \in E_{\lambda_{i}}:\left|x_{\lambda_{i}}\right| \leqq \sup \left[\sup _{i=1, \cdots, n}\left|B_{\lambda_{i}}\right|, n\right]\right\}, \quad i=1, \cdots, n
$$

and where $C_{\lambda}=(0)$ for all other $\lambda \in \Lambda$. Then $C$ is bounded in $E$ and 
the collection of such $C$ forms a bounded system for $E$.

Now we look at the dual of $E . E^{*}$ is the subspace of $\prod_{\lambda \in A} E_{\lambda}$ which consists of all $x^{*}=\left(x_{\lambda}^{*}\right)_{\lambda \in A}$ such that $x_{\lambda}^{*}=0$ except for a countable subset of indices $[7, p .81]$. We describe the neighborhoods of 0 in $E^{*}$. In the first place, let $C=\Pi_{\lambda \in A} C_{\lambda}$ be of the form just described. If $x^{*} \in(1 / n)\left[\left(\prod_{\lambda \in \Lambda} C_{\lambda}^{\prime}\right) \cap E^{*}\right]$, and if $y \in C$, then $\left|x^{*}(y)\right|=$ $\left|(1 / n) \sum_{i=1}^{n} x_{\lambda_{i}}^{*} y_{\lambda_{i}}\right| \leqq 1$, so that $x^{*} \in C^{\diamond}$. Next, assume that $x^{*} \in E^{*}$. but $x^{*} \notin \prod_{\lambda \in A} C_{\lambda^{\prime}}^{\prime}$. Then for some $\lambda_{0} \in \Lambda, x_{\lambda_{0}}^{*} \notin C_{\lambda_{0}}^{\prime}$ and thus $\left|x_{\lambda_{0}}^{*}\right|>1 /\left|C_{\lambda_{0}}\right|$. Let $y$ be defined by $y_{\lambda_{0}}=\left|C_{\lambda_{0}}\right|$ and $y_{\lambda}=0$ for all other $\lambda \in A$. Then $y \in C$ and $\left|x^{*}(y)\right|=\left|x_{\lambda_{0}}^{*}\right|\left|y_{\lambda_{0}}\right|>1$, so that $x^{*} \notin C^{\delta}$. Consequently $(1 / n)\left[\left(\Pi_{\lambda \in \Lambda} C_{\lambda}^{\prime}\right) \cap E^{*}\right] \subseteq C^{\dagger} \subseteq\left[\left(\Pi_{\lambda \in, 1} C_{\lambda}^{\prime}\right) \cap E^{*}\right]$, so that neighborhoods of 0 in $E^{*}$ contain neighborhoods of the form $\left(\Pi_{\lambda \in \Lambda} C_{\lambda}^{\prime}\right) \cap E^{*}$, which incidentally are closed in the dual topology on $E^{*}$ ! Consequently bounded sets in $E^{*}$ are contained in bounded sets of the form $\left(\prod_{\lambda \in \Lambda} B_{\lambda}^{*}\right) \cap E^{*}$, where for each $\lambda \in \Lambda, B_{\lambda}^{*}$ is bounded in $E_{\lambda}^{*}$. We finish the proof that $E$ is bi-lme by showing that the polar in $E$ of an arbitrary such set is idempotent. We may assume without loss of generality that each $B_{\lambda}^{*}$ is equilibrated and convex, and that $\left|B_{\lambda}^{*}\right| \geqq 1$. Now $x^{*} \in\left[\left(\prod_{\lambda \in \Lambda} B_{\lambda}^{*}\right) \cap E^{*}\right]$ implies that if $\left|x^{*}\right|=\left(\left|x_{\lambda}^{*}\right|\right)_{\lambda \in \Lambda}$, then $\left|x^{*}\right| \in\left[\left(\Pi_{\lambda \in \Lambda} B_{\lambda}^{*}\right) \cap E^{*}\right]$, so that if $y \in\left[\left(\Pi_{\lambda \in A} B_{\lambda}^{*}\right) \cap E^{*}\right]^{9}$, then $\left|x_{\lambda}^{*}\right|\left|y_{\lambda}\right| \leqq$ 1 for each $\lambda \in \Lambda$. In addition, if we let $\lambda_{0} \in \Lambda$ be arbitrary, and if we take $w^{*} \in\left(\prod_{\lambda \in A} B_{\lambda}^{*}\right) \cap E^{*}$ such that $w_{\lambda_{0}}^{*}=\left|B_{\lambda_{0}}^{*}\right|$ and $w_{\lambda}^{*}=0$ for all other $\lambda \in \Lambda$, then $z \in\left[\left(\Pi_{\lambda \in \Lambda} B_{\lambda}^{*}\right) \cap E^{*}\right]^{9}$ implies that $\left|w_{\lambda_{0}}^{*}\right|\left|z_{\lambda_{0}}\right|=$ $\left|w^{*}(z)\right| \leqq 1$, so that $\left|z_{\lambda_{0}}\right| \leqq 1 /\left|B_{\lambda_{0}}^{*}\right| \leqq 1$. From these last two statements, we see that if $x^{*} \in\left[\left(\prod_{\lambda \in A} B_{\lambda}^{*}\right) \cap E^{*}\right]$ and if $y, z \in\left[\left(\prod_{\lambda \in A} B_{\lambda}^{*}\right) \cap E^{*}\right]^{\rho}$, then

$$
\left|x^{*}(y z)\right| \leqq \sum_{\lambda \epsilon_{\Lambda}}\left|x_{\lambda}^{*}\right|\left|y_{\lambda}\right|\left|z_{\lambda}\right| \leqq \sum_{\lambda \epsilon_{\Lambda}}\left|x_{\lambda}^{*}\right|\left|y_{\lambda}\right|=\left|x^{*}\right|(|y|) \leqq 1,
$$

whereupon $y z \in\left[\left(\prod_{\lambda \in \Lambda} B_{\lambda}^{*}\right) \cap E^{*}\right]^{9}$. Consequently this last set is idempotent, which completes the proof that $E$, endowed with the strong topology, is lmc. We note in passing that if our $E$ has any Hausdorff lmc topology which gives it the above bounded system, then precisely the same argument shows that $E$ is bi-lme.

4. Associated sequences. The question which we ask in this section is whether or not there is any relationship between associated sequences for $E$ and those for $E^{* *}$. If the lmc algebra $E$ has $\left(E_{\lambda}\right)_{\lambda \in I}$ for an associated sequence, then we might hope that $\left\{\left(E_{\lambda}\right)^{* *}\right\}_{\lambda \in I}=$ $\left(E_{\lambda}^{* *}\right)_{\lambda \in \Lambda}$ would be an associated sequence for $E^{* *}$, in the sense that for each $\lambda \in \Lambda, E_{\lambda}^{* *} \cong\left(E^{* *}\right)_{\lambda}$ and $\left\{\left(E^{* *}\right)_{\lambda}\right\}_{\lambda \in \Lambda}$ forms an associated sequence for $E^{* *}$. Indeed, for $C[0,1)$, with pointwise multiplication and compact-open topology, there is an associated sequence the biduals of which form an associated sequence for $C^{* *}[0,1)$. To see that this is not always the case, we consider the following. 
EXAMPLE 4.1. Let $C(\Omega)$ be the algebra of continuous, complexvalued functions on the ordinals less than the first uncountable (with order topology), where the algebra is endowed with pointwise multiplication and compact-open topology. Then $C(\Omega)$ is an complete lme algebra [18, p. 13]; in fact $C(\Omega)$ is a bi-lme algebra. Inasmuch as the set $B=\{x \in C(\Omega):|x(t)| \leqq 1$, for all $t \in \Omega\}$ serves as a bounded system for $C(\Omega)\left[13\right.$, p. 75 , and 23, p. 274], $C^{*}(\Omega)$ is normed, so $B$ is a basis for $\left(C(\Omega), t_{b}\right)$. But $B$ is idempotent. Theorem 3.6 says that $C(\Omega)$ is then bi-lmc. Let $\left(S_{\lambda}\right)_{\lambda \in \Lambda}$ be a collection of compact subsets of $Q$ such that every compact subset of $Q$ is contained in at least one member of $\left(S_{\lambda}\right)_{\lambda \in \ell_{\lambda}}$. Then an associated sequence for $C(\Omega)$ can be taken to be of the form $\left\{C\left(S_{\lambda}\right)\right\}_{\lambda \in \lambda}$. From $\S 2$ we see that $C^{*}(\Omega)$ is a linear inductive limit of $\left\{C^{*}\left(S_{\lambda}\right)\right\}_{\lambda \in A}$. For each $\lambda \in \Lambda$, let $C^{\prime}\left(S_{\lambda}\right)$ be the subspace of $C^{*}(\Omega)$ which is linearly isomorphic to $C^{*}\left(S_{\lambda}\right)$, and put the $C^{*}\left(S_{\lambda}\right)$ topology on $C^{\prime}\left(S_{\lambda}\right)$. Since the topology of $C^{*}(\Omega)$, when restricted to $C^{\prime}\left(S_{\lambda}\right)$, coincides with the topology of $C^{\prime}\left(S_{\lambda}\right)$, it is apparent that $C^{* *}\left(S_{\lambda}\right)$ is equivalent to $C^{* *}(\Omega) /\left[C^{\prime}\left(S_{\lambda}\right)\right]^{\delta}$, where $C^{* *}(\Omega) /\left[C^{\prime}\left(S_{\lambda}\right)\right]^{b}$ has the quotient norm. However, $\left[C^{\prime}\left(S_{\lambda}\right)\right]^{\delta}$ contains a nontrivial subspace, so that on $C^{* *}(\Omega)$ the Minkowski functional $p_{\lambda}$ for $\left[C^{\prime}\left(S_{\lambda}\right)\right]^{d}$ is a seminorm, but not a norm. On the other hand, $C^{*}(\Omega)$ is a normed space, and $C^{* *}(\Omega)$ a Banach algebra, so the unit sphere of $C^{* *}(\Omega)$ has no nontrivial subspace. Thus the topology on $C^{* *}(\Omega)$ generated by the $\left(p_{\lambda}\right)_{\lambda \in 1}$ yields a topology on $C^{* *}(\Omega)$ weaker than the bidual topology. Consequently $\left\{C^{* *}\left(S_{\lambda}\right)\right\}_{\lambda \in 1}$ does not form an associated sequence for $C^{* * *}(\Omega)$.

Indeed, things are not always so bleak.

Lemma 4.2. Let $E$ be an lme algebra and let $E^{* *}$ have the Arens multiplication. Let $V$ be an idempotent neighborhood of 0 in $E$ and let $F^{*}$ be the subspace of $E^{*}$ spanned by $V^{\delta}$. Then $F^{* \delta}$ is a closed, two-sided ideal in $E^{* *}$.

Proof. $F^{* d}$ is closed in $E^{* *}$ because the polar of any set in $E^{*}$ is closed in $E^{* *}$. We prove now that $F^{* \delta}$ is a right ideal in $E^{* *}$. To this end let $x^{* *} \in F^{* \delta}$ and $y^{* *} \in E^{* *}$ and $z^{*} \in F^{*}$. We will show that $\left(x^{* *} \circ y^{* *}\right) z^{*}=0$. There is some finite $m$ such that $z^{*} \in m V^{\delta}$, since $z^{*} \in F^{*}$ and $V^{\circ}$ spans $F^{*}$. However, $V$ is idempotent, so that $\left(z^{*} \circ V\right) V=z^{*}(V \circ V) \subseteq z^{*}(V)$, whence $\left(z^{*} \circ V\right) \subseteq m V^{\circ}$, which means that $z^{*} \circ V$ is bounded in $E^{*}$. Now $y^{* *}$ is continuous on $E^{*}$, so sends bounded sets into bounded sets. Thus $\left(y^{* *} \circ z^{*}\right) V=y^{* *}\left(z^{*} \circ V\right)$ is bounded, so $\left(y^{* * \circ} \circ z^{*}\right) \in k V^{\delta} \subset F^{*}$ for some finite $k$. But $x^{* *} \in F^{* \downarrow}$, so $\left(x^{* * \circ} \circ y^{* *}\right) z^{*}=x^{* *}\left(y^{* *} \circ z^{*}\right)=0$, and consequently $\left(x^{* *} \circ y^{* *}\right) \in F^{* \delta}$ is a right ideal. To show that $F^{* \delta}$ is a left ideal we must show that 
$\left(y^{* *} \circ x^{* *}\right) z^{*}=0$. As above, $z^{*} \circ V \subseteq F^{*}$, so that $\left(x^{* *} \circ z^{*}\right) V=$ $x^{* *}\left(z^{*} \circ V\right)=0$. Since $V$ is an absorbing set in $E$, this implies that $x^{* *} \circ z^{*}=0$. Consequently $\left(y^{* * \circ} \circ x^{* *}\right) z^{*}=y^{* *}\left(x^{* *} \circ z^{*}\right)=0$, and $F^{* \delta}$ is a left ideal in $E^{* *}$.

THEOREM 4.3. Let $E$ be a nonnormable $F$ algebra with associated sequence $\left(E_{n}\right)_{n=1}^{\infty}$. If $E^{*}$ is an $L B$ space with defining sequence $\left\{\left(E_{n}\right)^{*}\right\}_{n=1}^{\infty}$, then $\left\{\left(E_{n}\right)^{* * *}\right\}_{n=1}^{\infty}$ is an associated sequence for $E^{* *}$.

Proof. Let $\left(E_{n}\right)^{*}$ and $\left(E_{n}\right)^{* *}$ be denoted $E_{n}^{*}$ and $E_{n}^{* *}$ respectively. Let $\left(V_{n}\right)_{n=1}^{\infty}$ be the basis for $E$ from which $\left(E_{n}\right)_{n=1}^{\infty}$ arises. Then $\left(V_{n}^{\delta} \delta\right)_{n=1}^{\infty}$ is a basis for $E^{* *}$. Let $p_{n}$ be the Minkowski functional semi-norm for $V_{n}^{\text {od }}$. Then $p_{n}^{-1}(0)$ is a closed ideal in $E^{* *}$. Since $E^{* *}$ is $F$ space [14, Corollary 3 to Theorem 1], the associated sequence of $E^{* *}$ corresponding to $\left(V_{n}^{\delta}{ }^{\delta}\right)_{n=1}^{\infty}$ has for members the completions of the algebras. $E^{* *} / p_{n}^{-1}(0)$ equipped with the quotient norm with respect to $p_{n}$, where the completion is in the norm topology. To complete the proof it suffices to show that for each $n, E_{n}^{* *} \cong E^{* *} / p_{n}^{-1}(0)$ algebraically and topologically. Note that this will mean $E^{* *} / p_{n}^{-1}(0)$ is complete. Let $n$ be fixed. Let $E_{n}^{\prime}$ be the subspace of $E^{*}$ which is linearly isomorphic to $E_{n}^{*}$, and let $E_{n}^{\prime}$ inherit its topology from $E_{n}^{*}$, so that $E_{n}^{\prime}$ and $E_{n}^{*}$ are naturally equivalent. That $E_{n}^{\prime}$ is topologically a subspace of $E^{*}$ follows from the fact that $E_{n}^{*}$ is a member of a defining sequence for the $L B$ space $E^{*}$. Now $p_{n}^{-1}(0)=E_{n}^{\prime \prime}$, so that $E^{* *} / p_{n}^{-1}(0)=E^{* *} / E_{n}^{\prime \prime}$. Next, since $E_{n}^{\prime} \cong E_{n}^{*}, E^{* *} / E_{n}^{\prime}$ is linearly isomorphic to $E_{n}^{* *}$, where $x^{* *} \in E_{n}^{\prime \delta}$ maps onto $x_{n}^{*}$, which is defined to be the element of $E_{n}^{* *}$ corresponding to the restriction of $x^{* *}$ to $E_{n}^{\prime}$. It is evident that the unit spheres of $E^{* *} / E_{n}^{\prime \delta}$ and $E_{n}^{* *}$ correspond to one another under the isomorphism, so that the two spaces are topologically isomorphic. We have left only the proof that the multiplications on the two spaces agree. By Lemma 4.2 we know that $E_{n}^{\prime b}$ is a closed ideal in $E^{* *}$, so that the multiplication in $E^{* *} / E_{n}^{\prime \prime}$ is defined by

$$
\left(x^{* *}+E_{n}^{\prime \prime}\right) \circ\left(y^{* *}+E_{n}^{\prime \prime}\right)=\left(x^{* * \circ} \circ y^{* *}\right)+E_{n}^{\prime \phi} \text {. }
$$

Let $x^{* *}, y^{* *} \in E^{* *}$, with $x_{n}^{* *}, y_{n}^{* *}$ the elements in $E_{n}^{* *}$ which correspond to the restrictions to $E_{n}^{\prime}$ of $x^{* *}$ and $y^{* *}$ respectively. Let "o" be the multiplication in $E_{n}$ obtained from $\circ$ in $E$. Next, let $z_{n}^{*} \in E_{n}^{*}$, and let $z_{n}^{\prime} \in E_{n}^{\prime}$ correspond to $z_{n}^{*}$. We wish to show that $\left(x_{n}^{* *} \circ_{n} y_{n}^{* *}\right) z_{n}^{*}=$ $\left(x^{* *} \circ y^{* *}\right) z_{n}^{\prime}$. For $w, v \in E$, with $w_{n}, v_{n}$ the corresponding elements in $E_{n}$, we see that $\left(z_{n}^{*} \circ_{n} w_{n}\right) v_{n}=\left(z_{n}^{\prime} \circ w\right) v$, so that $z_{n}^{*} \circ_{n} w_{n}$ in $E_{n}^{*}$ corresponds to $z_{n}^{\prime} \circ w$ in $E_{n}^{\prime} \subset E^{*}$. Since $y_{n}^{* *} \in E_{n}^{* *}$ corresponds to the restriction of $y^{* *}$ to $E_{n}^{\prime}$, it is immediate that for all $z_{n}^{*} \in E_{n}^{*}, y_{n}^{* *} \circ_{n} z_{n}^{*}$ in $E_{n}^{*}$ corresponds to $y^{* *} \circ z_{n}^{\prime}$ in $E_{n}^{\prime}$. Finally, by the same reasoning, 
$\left(x_{n}^{* *} \circ_{n} y_{n}^{* * \circ} y^{* *}\right) z_{n}^{*}=\left(x^{* *}\right) z_{n}^{\prime}$. But then the restriction to $E_{n}^{\prime}$ of $\left(x^{* *} \circ y^{* *}\right)$ in $E^{* *}$ corresponds to $x_{n}^{* *} \circ_{n} y_{n}^{* *}$ in the linear isomorphism between $E^{* *} / E_{n}^{\prime \delta}$ and $E_{n}^{* *}$, so the isomorphism preserves multiplication.

In the proof of Theorem 4.3 we did not use explicitly the metrizability of the $F$ algebra. What we need was that for each $n$, the topology of $E^{*}$, when restricted to the subspace $E_{n}^{\prime}$ coincided with the topology already on $E_{n}^{\prime}$ (defined to be equivalent to $E_{n}^{*}$ ), plus the fact that the bipolars of the members of a basis for $E$ formed a basis for the complete space $E^{* *}$. Thus we have

COROLLARY 4.4. Let $E$ be a complete quasi-barreled lme algebra such that $E^{* *}$ is complete, and let $E$ have $\left(E_{\lambda}\right)_{\lambda \in .1}$ as an associated sequence. Assume that for all $\lambda \in \Lambda$, the topology of $E^{*}$, when restricted to $E_{\lambda}^{\prime}$, coincides with the topology on $E_{\lambda}^{\prime}$. Then $\left\{\left(E_{\lambda}\right)^{* *}\right\}_{\lambda \in 1}$ is an associated sequence of $E^{* *}$.

An example in which the hypothesis of Theorem 4.3 holds is $C[0,1)$ with the compact-open topology. An associated sequence for $C[0,1)$ consists of $\{C[0,1-1 / n]\}_{n=1}^{\infty}$, where $C[0,1-1 / n]$ has supremum norm. It can be shown that the dual of $C[0,1)$ is an $L B$ space, with defining sequence $\left\{C^{*}[0,1-1 / n]\right\}_{n=1}^{\infty}$. Thus by Theorem 4.3 $\left\{C^{* *}[0,1-1 / n]\right\}_{n=1}^{\infty}$ forms and associated sequeuce for $C^{* *}[0,1)$. That Theorem 4.3 does not hold for all $F$ algebras comes from Amemiya's example [1] of an $F$ space (thus an $F$ algebra under zero multiplication) whose dual is not an $L B$ space.

Let $E$ be an $F$ algebra. In $E^{* *}$ we now confine our attention to associated sequences of the form $\left\{E^{* *} / p_{n}^{-1}(0)\right\}_{n=1}^{\infty}$, where $\left(p_{n}\right)_{n=1}^{\infty}$ is the collection of Minkowski functionals for the bipolars of the members of basis $\left(V_{n}\right)_{n=1}^{\infty}$ for $E$. We say that such a sequence is a restricted associated sequence for $E^{* *}$. We will give a necessary and sufficient condition for a nonnormable $F$ algebra $E$ with associated sequence $\left(E_{n}\right)_{n=1}^{\infty}$ to have $\left(E_{n}^{* *}\right)_{n=1}^{\infty}$ as a restricted associated sequence for $E^{* *}$.

Let $W$ be a neighborhood of 0 in $E$, and let $E_{W}^{\prime}$ be the vector subspace of $E^{*}$ spanned by $W^{\dot{\delta}}$. We can then give

Definition 4.5. A neighborhood $W$ of 0 in $E$ is smooth if and only if the function $h: E^{* *} \rightarrow E_{W}^{\prime *}=\left(E_{W}^{\prime}\right)^{*}$, defined for all $x^{* *} \in E^{* *}$ by $h\left(x^{* *}\right)=$ the restriction of $x^{* *}$ to $E_{W}^{\prime}$, maps onto $E_{W}^{\prime *}$.

We mention that a necessary and sufficient condition for $W$ to be smooth is that the Mackey topology $\tau\left(E^{*}, E^{* *}\right)$, when restricted to $E_{W}^{\prime}$, be stronger than the weak- $\left(E_{W}^{\prime *}\right)$ topology on $E_{W}^{\prime}$. For on the one hand, assume that the condition is satisfied, and let $x^{\prime *} \in E_{W}^{\prime *}$. Then $x^{\prime *}$ is weak- $\left(E^{\prime *}\right)$ continuous, so by hypothesis $x^{\prime *}$ is continuous for 
the restriction of $\tau\left(E^{*}, E^{* *}\right)$ to $E_{W}^{\prime}$. Thus by the Hahn-Banach theorem there is an extension of $x^{\prime *}$ to a continuous linear function on all of $E^{*}$. Evidently $h$ is then an onto map. On the other hand, let us assume that $W$ is smooth, and let $x^{\prime *} \in E_{W}^{\prime *}$. Then by assumption there is an $x^{* *} \in E^{* *}$ such that the restriction of $x^{* *}$ to $E_{W}^{\prime}$ is precisely $x^{\prime *}$. But then $x^{* *}$ is $\tau\left(E^{*}, E^{* *}\right)$ continuous. It follows directly that $\tau\left(E^{*}, E^{* *}\right)$ restricted to $E_{W}^{\prime}$ is stronger than the weak$\left(E^{\prime *}\right)$ topology on $E_{W}^{\prime}$.

THEOREM 4.6. Let $E$ be a nonnormable $F$ algebra with associated sequence $\left(E_{n}\right)_{n=1}^{\infty}$, where $\left(E_{n}\right)_{n=1}^{\infty}$ corresponds to the basis $\left(V_{n}\right)_{n=1}^{\infty}$ for $E$. Then $\left(E_{n}^{* *}\right)_{n=1}^{\infty}$ is a restricted associated sequence for $E^{* *}$ if and only if $V_{n}$ is smooth, for all $n$.

Proof. Assume that $V_{n}$ is smooth, for all $n$. Then the proof that $\left(E_{n}^{* *}\right)_{n=1}^{\infty}$ is an associated sequence for $E^{* *}$ is a repetition of the proof of Theorem 4.3, since in the proof of Theorem 4.3 we only needed the property of smoothness. To prove the other half of the theorem, assume that $\left(E_{n}^{* *}\right)_{n=1}^{\infty}$ is a restricted associated sequence for $E^{* *}$, so that for each $n$ we have $E^{* *} / E_{n}^{\prime \circ}=E^{* *} / p_{n}^{-1}(0) \cong E_{V_{n}}^{\prime *}=E_{n}^{\prime *}$, where the isomorphism takes $x^{* *}+E_{n}^{\prime \delta}$ into $x_{n}^{\prime *}$, which itself is the restriction of $x^{* *}$ to $E_{n}^{\prime}$. If we denote this isomorphism by $k_{n}$, then every $x_{n}^{\prime *} \in E_{n}^{\prime *}$ can be written as $k_{n}\left(x^{* *}+E_{n}^{\prime}\right)$, for some $x^{* *} \in E^{* *}$. But since $k_{n}\left(x^{* *}+E_{n}^{\prime}\right)=x^{* *}$ restricted to $E_{n}^{\prime}$, it follows that the map given in Definition 4.5 maps onto $E_{n}^{* *}$, whence $V_{n}$ is smooth.

We conclude $\S 4$ with the description of a new $F$ algebra, one which has topological properties of an $L_{1}$ space, and whose dual is an $L B$ space with defining sequence obtained from the $F$ algebra.

ExAmPle 4.7. For any $n \geqq 2$, let $A C_{n}=A C_{0}[0,1-1 / n]$ be the space of all complex-valued continuous functions on $[0,1-1 / n]$ which vanish at 0 and whose first derivatives exist and are absolutely integrable on $[0,1-1 / n]$. If we take the norm of a function in $A C_{n}$ to be its total variation on $[0,1-1 / n]$, then $A C_{n}$ is a Banach space, isometrically isomorphic to $L_{1}[0,1-1 / n]$ (see [12, p. 338]). With pointwise multiplication $A C_{n}$ becomes an algebra, an in fact a Banach algebra. For if $f$ and $g$ are in $A C_{n}$, then

$$
\begin{aligned}
\|(f g)\| & =\int_{0}^{1-1 / n}\left|(f g)^{\prime}(t)\right| d t \\
& \leqq \int_{0}^{1-1 / n}\left\{\left|f^{\prime}(t)\right||g(t)|+\left|g^{\prime}(t)\right||f(t)|\right\} d t \\
& \leqq \int_{0}^{1-1 / n}\left\{\left[\left|f^{\prime}(t)\right| \int_{0}^{t}\left|g^{\prime}(s)\right| d s\right]+\left[\left|g^{\prime}(t)\right| \int_{0}^{t}\left|f^{\prime}(s)\right| d s\right]\right\} d t
\end{aligned}
$$




$$
\leqq \int_{0}^{1-1 / n}\left|f^{\prime}(t)\right| d t \int_{0}^{1-1 / n}\left|g^{\prime}(t)\right| d t=\|f\|\|g\| \text {. }
$$

Now we transplant the multiplication of $A C_{n}$ onto $L_{1}[0,1-1 / n]$. Define the bilinear operation $\Delta_{n}$ on $L_{1}[0,1-1 / n]$ by

$$
\left(x_{n} \Delta_{n} y_{n}\right) t=x_{n}(t) \int_{0}^{t} y_{n}(s) d s+y_{n}(t) \int_{0}^{t} x_{n}(s) d s,
$$

for all $x_{n}, y_{n} \in L_{1}[0,1-1 / n]$, and almost all $t$ in $[0,1-1 / n]$. Then it is apparent that almost everywhere in $[0,1],\left(x_{n} \Delta_{n} y_{n}\right)$ is the function on $[0,1-1 / n]$ whose value at $t$ is the derivative of $\int_{0}^{t} x_{n}(s) d s \int_{0}^{t} y_{n}(s) d s$, so that the mapping from $A C_{n}$ to $L_{1}[0,1-1 / n]$ is an algebraic isomorphism. Consequently $\left(L_{1}[0,1-1 / n], \Delta_{n}\right)$ is a Banach algebra.

Next, let $I$ consist of all the functions defined on the half-open interval $[0,1)$ which vanish at 0 and which for all $n$ are absolutely integrable in $[0,1-1 / n]$. Since the function $x$ defined by $x(t)=$ $1 /(1-t)$ for all $t$ in $[0,1)$ is in $I$ but not in $L_{1}[0,1], I$ properly contains $L_{1}[0,1]$. Let a generating neighborhood system for $I$ be $\left(V_{n}\right)_{n=1}^{\infty}$, where for all $n, V_{n}=\left\{x \in I: \int_{0}^{1-1 / n}|x(t)| d t \leqq 1\right\}$. Then the topology on $I$ is metrizable, and $I$ is complete in this topology, since Cauchy sequences in $I$ converge pointwise (almost everywhere in $[0,1)$ ) to a function in $I$. Note that $I$ is nonnormable, because no neighborhood is bounded. The multiplication we put on $I$ is an extension of the multiplication given to $L_{1}[0,1-1 / n]$. Specifically, for $x, y \in I$, we define $x \Delta y$ by the equation $(x\lrcorner y) t=x(t) \int_{0}^{t} y(s) d s+y(t) \int_{0}^{t} x(s) d s$, for almost all $t$ in $[0,1)$. The different $J_{n}$ transmit the properties of closure, distributivity, and associativity to $\Delta$. That the unit sphere of $\left(L_{1}[0,1-1 / n], \Delta_{n}\right)$ is idempotent implies that $V_{n}$ is idempotent, for all $n$. Consequently $I$, with multiplication $\Delta$, is an $F$ algebra.

Let $p_{n}$ be the Minkowski functional for $V_{n}$. Then $I / p_{n}^{-1}(0)$ is linearly isomorphic to $L_{1}[0,1-1 / n]$. Since the characteristic function of $[0,1-1 / n]$ is in $I$, it is apparent that $I / p_{n}^{-1}(0)$, endowed with the quotient normed topology from $p_{n}$, is isometrically isomorphic to $L_{1}[0,1-1 / n]$. Because $p_{n}^{-1}(0)$ is a closed ideal in $I$, multiplication in $I / p_{n}^{-1}(0)$ is given by $\left(x+p_{n}^{-1}(0)\right) \Delta\left(y+p_{n}^{-1}(0)\right)=(x \Delta y)+p_{n}^{-1}(0)$, for all $x$ and $y$ in $I$. For any $z \in I$, let $z_{n}$ denote the restriction of $z$ to $[0,1-1 / n]$. Then $x, y \in I$ imply that $(x \Delta y)_{n}=x_{n} \Delta_{n} y_{n}$. Hence $I / p_{n}^{-1}(0)$ is equivalent to $\left(L_{1}[0,1-1 / n], \Delta_{n}\right)$ and an associated sequence for $I$ is $\left\{L_{1}[0,1-1 / n]\right\}_{n=2}^{\infty}$. Furthermore, for each $n, L_{1}[0,1-1 / n]$ is equivalent to the factor space $L_{1}[0,1-1 /(n+1)] / R_{n}$, where $R_{n}$ is the closed ideal in $L_{1}[0,1-1 /(n+1)]$ consisting of all the functions which vanish almost everywhere in $[0,1-1 / n]$. Consequently $L_{1}^{*}[0,1-1 / n]$ 
is linearly and topologically isomorphic to a proper subspace of $L_{1}^{*}[0,1-1 /(n+1)]$. One can prove that $I^{*}$ is an $L B$ space, so that the criterion of Theorem 4.3 is satisfied by $\left\{L_{1}^{*}[0,1-1 / n]\right\}_{n=2}^{\infty}$ which thus forms a (restricted) associated sequence of Banach algebras for $I^{* *}$. We will put this fact to use in $\S 6$.

5. Commutativity in the bidual of an LMC algebra. What are necessary and sufficient conditions on a commutative Banach algebra for its bidual to be commutative? Arens asked this question in 1950 . Although it is still open, progress has been made in $[3,4,8,10,15$, 20]. It was this particular question which led me to the general investigation of the biduals of lme algebras. I am indebted to Professor Bade for calling my attention to this commutativity problem, and for our discussions on it. In this section we concentrate on the question of when commutativity in an lme algebra is transmitted to its bidual.

Definition 5.1. Let $E$ be an lme algebra. Then $E$ is bicommutative if and only if as an algebra $E^{* *}$ is commutative.

THEOREM 5.2. Let $E=(E, \circ)$ be a complete lmc algebra with associated sequence $\left(E_{\lambda}\right)_{\lambda \in \Lambda}$. If for each $\lambda \in \Lambda, E_{\lambda}$ is bicommutative, then $E$ is also bicommutative.

Proof. Let $x^{* *}, y^{* *} \in E^{* *}$, and let $z^{\prime}$ be an arbitrary element in $E^{*}$. It suffices to prove that $\left(x^{* *} \circ y^{* *}\right) z^{\prime}=\left(y^{* *} \circ x^{* *}\right) z^{\prime}$. By the definition of $\circ, x^{* *} \circ z^{\prime}$ and $y^{* *} \circ z^{\prime}$ are elements of $E^{*}$. Consequently, since $E^{*}$ is an inductive limit of the $E_{\lambda}^{*}, x^{* *} \circ z^{\prime}$ and $y^{* *} \circ z^{\prime}$ and $z^{\prime}$ each correspond to an element in $E_{\lambda}^{*}$, for some $\lambda \in \Lambda$. Let $E_{\lambda}^{\prime}$ be the subspace of $E^{*}$ which is equivalent to $E_{\lambda}^{*}$, so that the topology of $E_{\lambda}^{\prime}$ is stronger than the restriction to $E_{\lambda}^{*}$ of the topology on $E^{*}$. Thus there exist elements $x_{\lambda}^{* *}, y_{\lambda}^{* *} \in E_{\lambda}^{* *}$ which correspond to the restrictions of $x^{* *}$ and $y^{* *}$ to $E_{\lambda}^{\prime}$. Furthermore, let $z_{\lambda}^{*}$ be the element in $E_{\lambda}^{*}$ corresponding to $z^{\prime}$ in $E_{\lambda}^{\prime}$. If we denote the multiplication in $E$ by " $\circ_{\lambda}$ " then exactly as in the latter part of the proof of Theorem 4.3, we find that $\left(x^{* *} \circ y^{* *}\right) z^{\prime}=\left(x_{\lambda}^{* *} \circ_{\lambda} y_{\lambda}^{* *}\right) z_{\lambda}^{*}$ and $\left(y^{* * \circ} \circ x^{* *}\right) z^{\prime}=\left(y_{\lambda}^{* *} \circ_{\lambda} x_{\lambda}^{* *}\right) z_{\lambda}^{*}$. Therefore

$$
\left(x^{* *} \circ y^{* *}\right) z^{\prime}=\left(x_{\lambda}^{*} \circ_{\lambda} y_{\lambda}^{* *}\right) z_{\lambda}^{*}=\left(y_{\lambda}^{* *} \circ_{\lambda} x_{\lambda}^{* *}\right) z_{\lambda}^{*}=\left(y^{* * \circ} x^{* *}\right) z^{\prime} .
$$

However, the $z^{\prime}$ in $E^{*}$ was arbitrary, so that $x^{* * \circ} y^{* *}=y^{* *} \circ x^{* *}$. Consequently $E^{* *}$ is bicommutative.

We mention that Theorem 5.2 does not depend on $E^{* *}$ being lmc. In [3] Arens proved that if $S$ is a compact, Hausdorff space and if $C(S)$ has the supremum, then $C(S)$ is bicommutative. If $T$ is an 
arbitrary completely regular topological space, and if $\mathscr{S}$ generates $T$ (in the sense of Michael [18, p. 76]), where each member of $\mathscr{S}$ is compact in $T$, then $C(T)$, under pointwise multiplication and with the topology of uniform convergence on the members of $\mathscr{S}$, is a complete lme algebra and $\{C(S)\}_{s \in \mathscr{S}}$ forms an associated sequence for $C(T)$. Consequently, by Arens' result and Theorem 5.2, $C(T)$ is bicommutative. The following theorem generalizes Corollary 6.3 of [8]:

Theorem 5.3. A subalgebra $F$ of a bicommutative algebra $E$ is also bicommutative.

Proof. It is well-known that $F^{*}$ is linearly isomorphic to $E^{*} / F^{\prime}$, but in general $F^{*}$ has a weaker topology (under the isomorphism) than does $E^{*} / F^{b}$. Consequently elements in $F^{* *}$ correspond to elements of $\left(E^{*} / F^{b}\right)^{*}$. But $\left(E^{*} / F^{b}\right)$ is linearly isomorphic to $F^{b} \dot{b}$ in $E^{* *}$. Therefore $F^{* *}$ is linearly isomorphic to a subspace of $F^{b}$ in $E^{* *}$, with the isomorphism given in the following way. For $x^{*}$ in $F^{*}$, let ext $x^{*}$ be any extension to $E$ of $x^{*}$. Then for $x^{* *} \in F^{* *}$, the function $X^{* *}$ on $E^{*}$ defined by $X^{* *}\left(\operatorname{ext} x^{*}\right)=x^{* *}\left(x^{*}\right)$ for all $x^{*} \in F^{*}$ is in $E^{* *}$, and the map $x^{* *} \rightarrow X^{* *}$ is a one-to-one, linear map of $F^{* *}$ into $F^{b}$. Let $x^{* *}, y^{* *} \in F^{* *}$, and let $X^{* *}$ and $Y^{* *}$ and $Y^{* *}$ be the corresponding elements in $F^{b} \delta \subseteq E^{* *}$. Let $x^{*}$ be an arbitrary element of $F^{*}$, and ext $x^{*}$ an extension of $x^{*}$ to $E$. If $z, w \in F$, then we have

$$
\left[\left(\operatorname{ext} x^{*}\right) \circ z\right] w=\left(\operatorname{ext} x^{*}\right)(z \circ w)=x^{*}(z \circ w)=\left(x^{*} \circ z\right) w,
$$

so that $\left(\operatorname{ext} x^{*}\right) \circ z=\operatorname{ext}\left(x^{*} \circ z\right)$ on $F$. Since $Y^{* *}$ is in $F^{\dot{\partial}}, Y^{* *}$ annihilates $F^{\prime}$, so that

$$
\left[\left(Y^{* *} \circ \operatorname{ext} x^{*}\right)\right] z=Y^{* *}\left[\operatorname{ext}\left(x^{*} \circ z\right)\right]=y^{* *}\left(x^{*} \circ z\right)=\left[\operatorname{ext}\left(y^{* * \circ} \circ x^{*}\right)\right] z,
$$

and hence on $F,\left(Y^{* *} \circ \operatorname{ext} x^{*}\right)=\operatorname{ext}\left(y^{* *} \circ x^{*}\right)$. Finally, since $X^{* *}$ also

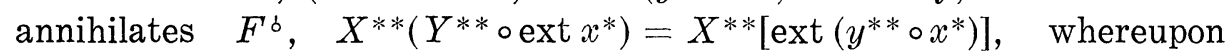

$$
\begin{aligned}
\left(x^{* *} \circ y^{* *}\right) x^{*} & =x^{* *}\left(y^{* *} \circ x^{*}\right)=X^{* *}\left[\operatorname{ext}\left(y^{* *} \circ x^{*}\right)\right] \\
& =X^{* *}\left(Y^{* *} \circ \operatorname{ext}\left(x^{*}\right)\right)=\left(X^{* *} \circ Y^{* *}\right) \operatorname{ext} x^{*} \\
& =\left(Y^{* *} \circ X^{* *}\right) \operatorname{ext} x^{*}=\cdots=\left(y^{* *} \circ x^{* *}\right) x^{*} .
\end{aligned}
$$

Therefore $x^{* *} \circ y^{* *}=y^{* *} \circ x^{* *}$ and $F$ is bicommutative.

We now investigate what happens to the bicommutativity of an Ime algebra when one makes certain changes in multiplication.

THEOREM 5.4. Let $p$ be a continuous, linear operator on the bicommutative lmc algebra $E=(E, \circ)$. Define the bilinear operation \lrcorner on $E$ by $x \Delta y=x \circ p(y)+p(x) \circ y$ for all $x$ and $y$ in $E$. If $\Delta$ is 
associative, so that $(E, \Delta)$ is an lmc algebra, then $(E, \Delta)$ is bicommutative and for all

$$
x^{* *}, y^{* *} \in E^{* *}, x^{*} \Delta y^{* *}=x^{* *} \circ p^{* *}\left(y^{* *}\right)+p^{* *}\left(x^{* *}\right) \circ y^{* *} .
$$

Proof. That $p^{* *}$, the second adjoint of $p$, is linear and continuous is well-known and easy to prove. Let $x, y \in E$, and $x^{*} \in E^{*}$. Then

$$
\begin{aligned}
(\hat{x} \Delta \hat{y}) x^{*} & =x^{*}(x \Delta y)=x^{*}\left([x \circ p(y)+p(x) \circ y]=\left[x^{*} \circ x\right] p(y)+\left[\hat{y} \circ x^{*}\right] p(x)\right. \\
& =p^{* *}(\hat{y})\left[x^{*} \circ x\right]+p^{* *}(\hat{x})\left[\hat{y} \circ x^{*}\right]=\left\{\hat{x} \circ p^{* *}(\hat{y})+p^{* *}(\hat{x}) \circ \hat{y}\right\} x^{*} .
\end{aligned}
$$

Thus $\hat{x} \Delta \hat{y}=\hat{x} \circ p^{* *}(\hat{y})+\hat{y} \circ p^{* *}(\hat{x})$. Next, let $y^{* *} \in E^{* *}$ and $x \in E$. There is a net $\left(\hat{y}_{\alpha}\right)_{\alpha} \in_{A}$ which converges weak- $\left(E^{*}\right)$ to $y^{* *}$, so that by Lemma 3.4 (ii), $\left\{\left(\widehat{x} \Delta \hat{y}_{\alpha}\right)\right\}_{\alpha \in A}$ converges weak- $\left(E^{*}\right)$ to $\hat{x} \Delta y^{* *}$. On the other hand, for any $x^{*}$ in $E^{*}$,

$$
\begin{aligned}
\left(\hat{x} \Delta \hat{y}_{\alpha}\right) x^{*} & =\left[p^{* *}\left(\hat{y}_{\alpha}\right)\right]\left(x^{*} \circ x\right)+\hat{y}_{\alpha}\left[x^{*} \circ p(x)\right] \\
& =\hat{y}_{\alpha}\left\{\left[p^{*}\left(x^{*} \circ x\right)+x^{*} \circ p(x)\right]\right\} \underset{\alpha}{\rightarrow} y^{* *}\left[p^{*}\left(x^{*} \circ x\right)+x^{*} \circ p(x)\right] \\
& =\left[\hat{x} \circ p^{* *}\left(y^{* *}\right)+p^{* *}(\hat{x}) \circ y^{* *}\right] x^{*} .
\end{aligned}
$$

Thus $\hat{x} \Delta y^{* *}=\hat{x} \circ p^{* *}\left(y^{* *}\right)+p^{* *}(\hat{x}) \circ y^{* *}$. Now let $x^{* *}, y^{* *} \in E^{* *}$. Again there is a net $\left(\widehat{x}_{\beta}\right)_{\beta \in_{B}}$ which converges weak- $\left(E^{*}\right)$ to $x^{* *}$. By Lemma $3.4(\mathrm{i}),\left\{\left(\hat{x}_{\beta} \Delta y^{* *}\right)\right\}_{\beta \in_{B}}$ converges weak-( $\left.E^{*}\right)$ to $x^{* *} \Delta y^{* *}$. If $x^{*} \in E^{*}$, then

$$
\begin{aligned}
\left(\widehat{x}_{\beta} \Delta y^{* *}\right) x^{*} & =\widehat{x}_{\beta}\left\{\left[p^{* *}\left(y^{* *}\right) \circ x^{*}\right]+\left[p^{*}\left(y^{* *} \circ x^{*}\right)\right]\right\} \rightarrow x^{* *}\left\{\left[p^{* *}\left(y^{* *}\right) \circ x^{*}\right]\right. \\
& \left.\left.+\left[p^{*}\left(y^{* *} \circ x^{*}\right)\right]\right\}=\left\{\left[x^{* *} \circ p^{* *}\left(y^{* *}\right)\right]+p^{* *}\left(x^{* *}\right) \circ y^{* *}\right]\right\} .
\end{aligned}
$$

Consequently $x^{* *} \Delta y^{* *}=x^{* *} \circ p^{* *}\left(y^{* *}\right)+p^{* *}\left(x^{* *}\right) \circ y^{* *}$ for all $x^{* *}$, $y^{* *} \in E^{* *}$, and since $(E, \circ)$ is bicommutative, the formula for $\Delta$ in $E^{* *}$ shows that $(E, \Delta)$ is also bicommutative.

If $E$ is an lmc algebra, then the maximal ideal space of $E$, denoted by $\Phi_{E}$, consists of all complex-valued continuous multiplicative linear functionals on $E$. For properties of the maximal ideal spaces of Banach algebras and lme algebras consult $[18,19]$.

THEOREM 5.5. Let $E_{1}=(E, \circ)$ and $E_{2}=(E, \Delta)$ be semi-simple, commutative, complete lmc algebras with the same closed regular maximal ideals. Assume that $E_{1}$ has an identity element $e_{1}$. If $E_{1}$ is bicommutative, then $E_{2}$ is also bicommutative, and $x \Delta y=\left(e_{1} \Delta e_{1}\right) \circ x \circ y$ for all $x, y \in E$.

Proof. Let the maximal ideal space of $E_{1}$ and $E_{2}$ be $\Phi_{E_{1}}$ and $\Phi_{E_{2}}$ respectively. If $f_{1}$ is in $\Phi_{E_{1}}$, then there is an $f_{2}$ in $\Phi_{E_{2}}$ and a nonzero complex number $a$ such that $f_{1}=a f_{2}$. Let $x, y \in E$. Then 


$$
f_{1}(x \Delta y)=a f_{2}(x \Delta y)=a f_{2}(x) f_{2}(y)=(1 / a) f_{1}(x) f_{1}(y)=(1 / a) f_{1}(x \circ y) .
$$

Now for $x=y=e_{1}, f_{1}\left(e_{1} \Delta e_{1}\right)=(1 / a) f_{1}\left(e_{1} \circ e_{1}\right)=1 / a$. Thus, $f_{1}(x \Delta y)=$ $f_{1}\left[\left(e_{1} \Delta e_{1}\right) \circ x \circ y\right]$, so the semi-simplicity of $E_{1}$ yields that $x \Delta y=$ $\left(e_{1} \Delta e_{1}\right) \circ x \circ y$. Multiplication in $E_{1}$ is continuous, so if $p: E \rightarrow E$ is defined by $\mathrm{p}(x)=\left(e_{1} \Delta e_{1}\right) \circ x$, for all $x \in E$, then $p$ is a continuous linear operator on $E$. Now mimic the proof of Theorem 5.4 to show that $E_{2}$ is bicommutative.

Definition 5.6. An algebra $E$ is factorable if and only if for $x \in E$, there exist $y, z \in E$ such that $x=y z$.

In [9], P. J. Cohen showed that if $E$ is a Banach algebra with left approximate identity, then $E$ is factorable. As our final result in $\S 5$ we have

Theorem 5.7. Let $F=(E$, o) and $G=(E, \Delta)$ be semi-simple, commutative $F$ algebras with the same closed regular maximal ideals. Assume that $F$ is factorable and bicommutative. Then $G$ is bicommutative.

Proof. Part of the proof is patterned after a portion of the proof of Theorem 5 in [5]. Let the Gelfand representation of $F$ be denoted by $\widetilde{F}$. Let elements of $\widetilde{F}$ be of the form $\widetilde{x}$. By the proof of Theorem 5.5, for any nonzero $f_{1}$ in $\Phi_{F}$, there is an $f_{2}$ in $\Phi_{G}$ and a nonzero complex number $a_{f_{1}}$ such that $f_{2}=a_{f_{1}} f_{1}$, so that for $x, y \in F, f_{1}(x \Delta y)=a_{f_{1}} f_{1}(x \circ y)$, whence $(\widetilde{x \Delta y}) f_{1}=a_{f_{1}}(\widetilde{x} \circ \widetilde{y}) f_{1}$. Let $g$ be defined on $\Phi_{F}$ by $g\left(f_{1}\right)=a_{f_{1}}$, for all $f_{1} \in \Phi_{F}$. Then $g \circ \widetilde{x} \circ \widetilde{y}=\widetilde{x \Delta y}$, for all $\mathrm{x}, y \in F$. Next, define the operator $h$ on $F$ by $h(x)=y$ if and only if $g \circ \widetilde{x}=\widetilde{y}$. Then $\widetilde{h(x)}=$ $g \circ \widetilde{x}$ for all $x \in F$. We must prove that $h$ is well-defined. Since $F$ is factorable, for any $x \in F$ there are $y, z \in F$ such that $x=y \circ z$. Then $\widetilde{h(x)}=g \circ \widetilde{x}=g \circ \widetilde{y} \circ z=\widetilde{y \Delta z}$, so that since $F$ is semi-simple, $h(x)=y \Delta z$. Thus $h$ is defined and uniquely so on $F$. Because $F$ is semisimple, $h$ is linear. To show that $h$ is continuous, assume that $\left[\left(x_{n}, h\left(x_{n}\right)\right]_{n=1}^{\infty}\right.$ converges to $(w, z)$ in the product metric of the product space $F \times F$. Then clearly $\left(x_{n}\right)_{n-1}^{\infty}$ converges to $w$ in the topology of $F$. To show that $h(w)=z$, let $f_{1}$ be an arbitrary element of $\Phi_{F}$. Since $f_{1}$ is continuous, $\lim \widetilde{x}_{n}\left(f_{1}\right)=\lim f_{1}\left(x_{n}\right)=f_{1}(w)=\widetilde{w}\left(f_{1}\right)$. Consequently $\lim \left(g \circ \widetilde{x}_{n}\right) f_{1}=\lim ^{n}\left[g\left(f_{1}\right) \widetilde{x}_{n}\left(f_{1}\right)\right]^{n}=g\left(f_{1}\right) \lim \widetilde{x}_{n}\left(f_{1}\right)=g\left(f_{1}\right) \tilde{w}\left(f_{1}\right)=$ $(g \circ \widetilde{w}) f_{1}$. On the other hand, by assumption $\left\{h\left(x_{n}\right)\right\}_{n=1}^{\infty}$ converges in the metric of $F$ to $z$, so that $\lim _{n} f_{1}\left[h\left(x_{n}\right)\right]=f_{1}(z)$. This means that $\widetilde{h(w) f_{1}}=(g \circ \widetilde{w}) f_{1}=\lim \left(g \circ \widetilde{x}_{n}\right) f_{1}=\lim \left[\widetilde{h\left(x_{n}\right)}\right] f_{1}=\widetilde{z}\left(f_{1}\right)$, and since $F$ is semi-simple and $f_{1}$ is an arbitrary element of $\Phi_{F}$, we conclude that 
$h(w)=z$. By the closed graph theorem, then, $h$ is continuous. If for some $x, y \in F$ we had $x \Delta y \neq x \circ[h(y)]$, then $\widetilde{x \Delta y} \neq x \circ \widetilde{\circ}(y)]=$ $\tilde{x} \circ[\widetilde{h(y)}]=\tilde{x} \circ g \circ \tilde{y}=g \circ \tilde{x} \circ \tilde{y}=\widetilde{x \Delta y}$, a contradiction. Therefore $x \Delta y=$ $x \circ[h(y)]$ for all $x, y \in F$. Now if we use the argument in the proof of Theorem 5.4, it is apparent that whenever $F$ is bicommutative, $G$ is also bicommutative.

6. Two examples. Kamowitz proved in [15] that if $X$ is a compact, Hausdorff space, then any multiplication on $C(X)$ which makes it into a commutative Banach algebra under the supremum norm makes $C(X)$ a bicommutative algebra. The first example in $\S 6$ is, then, known to be bicommutative. However, we give a new proof of that fact, based on Theorems 5.3 and 5.4. Civin and Yood [8] displayed a condition which assures one of a nonzero radical in the bidual of a Banach algebra. In our first example we show that the bidual of our Banach algebra has an infinite-dimensional radical, even though Civin and Yood's condition is not met. Our second example is a nonnormable $F$ algebra which is not bicommutative, and whose bidual has a nonzero radical.

EXAmple 6.1. Let $E$ be the Banach space of all continuous functions on $[0,1]$ which vanish at 0 , with norm equal to twice the supremum. Let the multiplication be given in the following way. For $x, y \in E$, and $t \in[0,1]$, define $\Delta$ by

$$
(x \Delta y) t=x(t) \int_{0}^{t} y(s) d s+y(t) \int_{0}^{t} x(s) d s .
$$

Then $E$ is equivalent to a subalgebra of the Banach algebra of all continuous, complex-valued functions on $[0,1]$ which vanish at 0 and which have continuous first derivatives on $[0,1]$, with pointwise multiplication and norm which is equal to the sum of the sup norm and the sup of the first derivative, as is shown in [12, p. 344]. It is easy to show that $E$ is a Banach algebra; we omit the proof. If we define $p: E \rightarrow E$ by $p(x) t=\int_{0}^{t} x(s) d s$ for $t$ in $[0,1]$, then $p$ is a linear, continuous function on $E$, and $x \Delta y=x \circ p(y)+y \circ p(x)$, where $\circ$ represents pointwise multiplication on $C[0,1]$. Consequently, by Theorems 5.3 and 5.4, $E$ is bicommutative. In order to show that $E^{* *}$ has an infinite-dimensional radical, let $t$ be fixed, momentarily, in $(0,1]$. Then let $\left(x_{n}\right)_{n=1}^{\infty}$ be a sequence in $E$ with the following properties: $0 \leqq x_{n} \leqq 1$, $x_{n}(t)=1$, and $x_{n}(s)=0$ whenever $|s-t| \geqq 1 / 2 n$. Let $x^{*} \in E^{*}$, so that by the Riesz-Kakutani theorem there is a regular, countably additive measure $m_{x^{*}}$ on $(0,1]$ corresponding to $x^{*}$. Then 


$$
\begin{aligned}
{\left[p^{*}\left(x^{*}\right)\right] x_{n}=x^{*}\left[p\left(x_{n}\right)\right] } & =\int_{0}^{1}\left[\int_{0}^{t} x_{n}(s) d s\right] m_{x^{*}}(d t) \\
& \leqq \int_{0}^{1}(1 / n) m_{x^{*}}(d t) \leqq 2\left\|x^{*}\right\|(1 / n)
\end{aligned}
$$

so that $\liminf _{n \rightarrow \infty}\left[p^{*}\left(x^{*}\right)\right] x_{n}=0$. But for each $n, \quad\left(x_{t}^{*}\right) x_{n}=x_{n}(t)=1$, where $x_{t}^{*}$ corresponds to the point mass of weight 1 at the point $t$. Hence $\left\|p^{*}\left(x^{*}\right)-x_{t}^{*}\right\| \geqq 1 / 2$, and $x_{t}^{*} \notin p^{*}\left(E^{*}\right)$. Now if we let $F$ be the closed linear span of the union of $p^{*}\left(E^{*}\right)$ and $\left\{x_{s}^{*}: s \in(0,1]\right.$ with $s \neq t\}$, then it is clear that $x_{t}^{*} \notin F$, since $\lim \inf x_{s}^{*}\left(x_{n}\right)=0$ for each $s \neq t$ and $x_{t}^{*}\left(x_{n}\right)=1$ and since $x_{t}^{*} \notin p^{*}\left(E^{*}\right)$. By the Hahn-Banach theorem there exists a nonzero $x^{* *} \in E^{* *}$ such that $x^{* *}$ is zero on $F$ and 1 on $x_{t}^{*}$. Consequently for any $x^{*} \in E^{*}$, we have

$$
\left(x^{* *}-1 x^{* *}\right) x^{*}=\left[p^{* *}\left(x^{* *}\right) \circ 2 x^{* *}\right] x^{*}=x^{* *}\left[p^{*}\left(2 x^{* *} \circ x^{*}\right)\right]=0,
$$

whence $x^{* *}$ is nilpotent, and is in the radical of $E^{* *}$. Furthermore, for different $t$ in $(0,1]$ the associated $x^{* *}$ are linearly independent from one another, by construction. Thus the radical of $E^{* *}$ is infinitedimensional. Finally, to see that the method of proof of Theorem 3.12 in [8] is not applicable, we show that the linear span of $\left(E^{*} \Delta E\right)$ is dense in $E^{*}$. To that end let $e>0$ and take an arbitrary $x^{*} \in E^{*}$, with $m_{x^{*}}$ the corresponding regular, countably additive measure on $(0,1]$. There exists an $n$ such that $\left|m_{x^{*}}\right|(0,1 / n] \leqq e / 2$, where $\left|m_{x^{*}}\right|$ is the total variation measure of $m_{x^{*}}$. For each positive $n$, let $y_{n}$ be defined by

$$
y_{n}(t)=\left\{\begin{array}{l}
4 n^{2} t, \quad 0 \leqq t<1 / 2 n \\
4 n-4 n^{2} t, \quad 1 / 2 n \leqq t<1 / n \\
0, \quad 1 / n \leqq t \leqq 1
\end{array}\right\}
$$

Note that for $t \geqq 1 / n, \quad \int_{0}^{t} y_{n}(s) d s=1$. Then for any $z \in E$ with $\|z\| \leqq 1$ we have

$$
\begin{aligned}
\left|\left[\left(x^{*} \Delta y_{n}\right)-x^{*}\right] z\right| & \leqq \int_{0}^{1}\left|\left[y_{n}(t) \int_{0}^{t} z(s) d s+z(t) \int_{0}^{t} y_{n}(s) d s\right]-z(t)\right|\left|m_{x^{*}}\right|(d t) \\
& \leqq 2 n[\|z\| / 2 n] \int_{0}^{1 / n}\left|m_{x^{*}}\right|(d t)+\|z / 2\| \int_{0}^{1 / n}\left|m_{x^{*}}\right|(d t) \leqq e
\end{aligned}
$$

Thus the linear span of $\left(E^{*} \Delta E\right)$ is dense in $E^{*}$, which is what we set out to prove.

ExAMPLE 6.2. Let $E$ denote $L_{1}[0,1]$ with multiplication like that described in Example 4.7: $x, y \in E$ implies that 


$$
(x \Delta y) t=x(t) \int_{0}^{t} y(s) d s+y(t) \int_{0}^{t} x(s) d s
$$

for almost all $t$ in $[0,1]$. We first show that $E$ is not bicommutative. Sills [20] proved it by direct computation; our method utilizes results of [8]. For each positive $n$, let $e_{n}$ be defined on $[0,1]$ by the equations

$$
e_{n}(t)=\left\{\begin{array}{cc}
n, & 0 \leqq t<1 / n \\
0, & 1 / n \leqq t \leqq 1
\end{array}\right\} .
$$

Then $\left\|e_{n}\right\|=1$, for each $n$. Let $x^{*} \in E^{*}$, and let $h_{x^{*}} \in L_{\infty}[0,1]$ be the function which corresponds to $x^{*}$ in the natural isometric isomorphism between the two spaces [12, p. 289]. Then for $x \in E$ we have

$$
\begin{aligned}
\mid x^{*}\left(x \Delta e_{n}\right) & -x^{*}(x)|=| \int_{0}^{1} h_{x^{*}}(t)\left[x(t) \int_{0}^{t} e_{n}(s) d s+e_{n}(t) \int_{0}^{t} x(s) d s\right] d t \\
& -\int_{0}^{1} h_{x^{*}}(s) x(s) d s\left|\leqq \int_{0}^{1}\right| h_{x^{*}}(t) \mid\left\{|x(t)|\left[1-\int_{0}^{t} e_{n}(s) d s\right]\right\} d t \\
& +\int_{0}^{1}\left|h_{x^{*}}(t)\right| e_{n}(t)\left[\int_{0}^{t}|x(s)| d s\right] d t \\
& =\int_{0}^{1 / n}\left|h_{x^{*}}(t)\right|\left\{|x(t)|\left[1-\int_{0}^{t} e_{n}(s) d s\right]\right\} d t \\
& +\int_{0}^{1 / n}\left|h_{x^{*}}(t)\right| e_{n}(t)\left[\int_{0}^{t}|x(s)| d s\right] d t \rightarrow 0
\end{aligned}
$$

as $n$ gets large. Consequently $\left(e_{n}\right)_{n=1}^{\infty}$ is a weak- $\left(E^{*}\right)$ right identity for $E$, in the sense that for each $x^{*} \in E^{*}$, and each $x \in E$, $\lim x^{*}\left(x \Delta e_{n}\right)=$ $x^{*}(x)$. Next we show that the linear span of $\left(E^{*} \Delta E\right)$ is not dense in $E^{*}$. To this end, let $x^{*} \in E^{*}$, and $x, y \in E$, and let $h_{x^{*}} \in L_{\infty}[0,1]$ correspond to $x^{*}$. By Fubini's theorem we have

$$
\begin{aligned}
\left(x^{*} \Delta x\right) y & =\int_{0}^{1} h_{x^{*}}(t)(x \Delta y)(t) d t \\
& =\int_{0}^{1} h_{x^{*}}(t)\left[x(t) \int_{0}^{t} y(s) d s+y(t) \int_{0}^{t} x(s) d s\right] d t \\
& =\int_{0}^{1} y(t)\left[h_{x^{*}}(t) \int_{0}^{t} x(s) d s\right] d t+\int_{0}^{1} y(t)\left[\int_{t}^{1} x(s) h_{x^{*}}(s) d s\right] d t \\
& =\int_{0}^{1} y(t)\left[h_{x^{*}}(t) \int_{0}^{t} x(s) d s+\int_{t}^{1} x(s) h_{x^{*}}(s) d s\right] d t .
\end{aligned}
$$

Consequently, for almost all $t \in[0,1]$,

$$
h_{x^{*} s_{x}}(t)=h_{x^{*}}(t) \int_{0}^{t} x(s) d s+\int_{t}^{1} x(s) h_{x^{*}}(s) d s .
$$

Now $\lim _{t \rightarrow 0} \int_{0}^{t} x(s) d s=0$, and $h_{x^{*}}$ is essentially bounded on $[0,1]$, and 
furthermore, $\int_{t}^{1} x(s) h_{x^{*}}(s) d s$ is continuous as a function of $t$ in $[0,1]$, so that $\lim _{t \rightarrow 0} h_{\left.x^{*}\right\lrcorner x}(t)=\int_{0}^{1} x(s) h_{x^{*}}(s) d s$, which just says that $h_{x^{*} \Delta x}$ is continuous at 0 . On the other hand, let $h_{0}$ be defined by

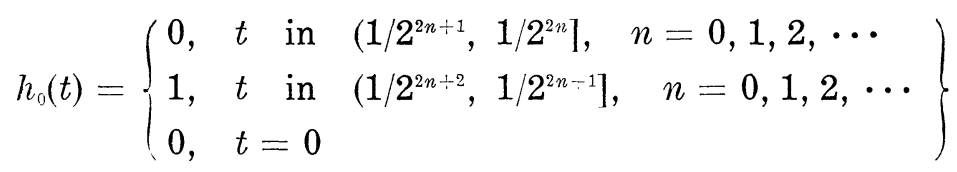

Then $h_{0}$ is in $L_{\infty}[0,1]$, but $\left\|h_{0}-h_{\left.x^{*}\right\lrcorner x}\right\| \geqq 1 / 2$ in the $L_{\infty}[0,1]$ norm, for each $x^{*} \in E^{*}$ and each $x \in E$. Thus the linear span of $E^{*} \Delta E$ is not dense in $E^{*}$. By the Hahn-Banach theorem, there is a nonzero $y^{* *} \in E^{* *}$ which vanishes on $E^{*} \Delta E$. Since $\left(e_{n}\right)_{n=1}^{\infty}$ is a weak- $\left(E^{*}\right) \operatorname{right}$ identity for $E$, Lemma 3.8 of [8] tells us that $E^{* *}$ has a right identity $e^{* *}$. Therefore $y^{* *} \Delta e^{* *}=y^{* *} \neq 0$. On the other hand, since $y^{* *}$ vanishes on $E^{*} \Delta E, y^{* *} \Delta x^{*}=0$ for each $x^{*} \in E^{*}$. Consequently, for any $x^{* *} \in E^{* *},\left(x^{* *} \Delta y^{* *}\right) x^{*}=x^{* *}\left(y^{* *} \Delta x^{*}\right)=0$ for every $x^{*} \in E^{*}$, whereupon $x^{* *} \Delta y^{* *}=0$. In particular, $e^{* *} \Delta y^{* *}=0$. Thus $E^{* *}$ is not commutative. Incidentally, since the right multiplication by $y^{* *}$ is the zero operator, $y^{* *}$ is in the radical of $E^{* *}$. It is apparent that $I$ is not bicommutative, where $I$ is the $F$ algebra with associated sequence $\left.\left\{L_{1}[0,1-1 / n], \Delta_{n}\right)\right\}_{n=2}^{\infty}$ defined in Example 4.7. For $\left\{\left(L_{1}^{* *}[0,1-1 / n]\right.\right.$, $\left.\left.\Delta_{n}\right)\right\}_{n=2}^{\infty}$ is an associated sequence for $I^{* *}$. Finally, we can easily show that $I^{* *}$ is not semi-simple. Indeed, if the $h_{0}$ defined above is restricted to the interval $[0,1 / 2]$, then the restriction is in $I^{*}$, and is not in the closure of the linear span of $I^{*} \Delta I$. Then, as above, the Hahn-Banach theorem yields a nonzero $z^{* *} \in I^{* *}$ which vanishes on $I^{*} \Delta I$. Consequently $z^{* *} \Delta I^{* *}=0$, so $z^{* *}$ is in the radical of $I^{* *}$.

We comment first that Example 6.2 shows that the Banach algebra of absolutely continuous functions on $[0,1]$ is nonbicommutative, and its bidual has a nonzero radical. Secondly, one cannot say that $I^{\text {** }}$ is not semi-simple just because the members of a particular associated sequence are nonsemi-simple.

The author would like to express his appreciation to Professors Duane W. Bailey and Cassius Ionescu Tulcea, and especially to Professors Charles E. Rickart and William G. Bade for their kind assistance in its preparation.

\section{BIBLIOGRAPHY}

1. I. Amemiya, Some examples of $(F)$ and $(D F)$ spaces, Proc. Japan Acad. 33 (1957), 169-171.

2. R. F. Arens, A generalization of normed rings, Pacific J. Math. 2 (1952), 455-471.

3. - Operations induced in function classes, Monatshefte für Math. u. Physik.

55 (1951), 1-19. 
4. R. F. Arens, The adjoint of a bilinear operation, Proc. Amer. Math. Soc. 2 (1951), 839-848.

5. F. I. Birtel, Banach algebras of multipliers, Duke Math. J. 28 (1961), 203-212.

6. N. Bourbaki, Espaces vectoriels topologiques, Chaps. I-II, Act. Sci. et Ind. 1189 (1953).

7. N. Bourbaki, Espaces vectoriels topologiques, Chaps. III-V, Act. Sci. et Ind. 1229 (1955).

8. P. Civin and B. Yood, The second conjugate space of a Banach algebra as an algebra, Pacific J. Math. 11 (1961), 847-870.

9. P. J. Cohen, Factorization in group algebras, Duke Math. J. 26 (1959), 199-205.

10. M. M. Day, Amenable semigroups, Illinois J. of Math. 1 (1957), 509-544.

11. J. A. Dieudonné and L. Schwartz, La dualité dans les espaces $(F)$ et $(L F)$, Ann. Inst. Fourier, Grenoble 1 (1950), 61-101.

12. N. Dunford and J. T. Schwartz, Linear Operators, Part I: General Theory, Interscience, New Nork (1958).

13. L. Gillman and M. Jerison, Rings of Continuous Functions, Van Nostrand, Princeton (1960).

14. A. Grothendieck, Sur les espaces $(F)$ et $(D F)$, Summa Brasil. Math. 3 (1954), $57-123$.

15. H. M. Kamowitz, Cohomology groups of commutative Banach algebras, Trans. Amer. Soc. 102 (1962), 352-372.

16. J. L. Kelley and I. Namioka and co-authors, Linear Topological Spaces, Van Nostrand, New York, (1963).

17. G. M. Köthe, Topologische Lineare Räume, Part I, Springer, Berlin (1960).

18. E. A. Michael, Locally multiplicatively-convex topological algebras, Memoirs Amer. Math. Soc. 11 (1952).

19. C. E. Rickart, General Theory of Banach Algebras, Van Nostrand, New York (1960).

20. W. H. Sills, Dissertation at the University of California, Berkeley (1963).

21. S. L. Warner, Inductive limits of normed algebras, Trans. Amer. Math. Soc. 82 (1956), 190-216.

22. - Weak locally multiplicatively-convex algebras, Pacific J. Math. 5, Suppl. 2 (1955), 1025-1032.

23. - The topology of compact convergence on continuous function spaces, Duke Math. J. 25 (1958), 265-282. 


\section{PACIFIC JOURNAL OF MATHEMATICS}

\section{EDITORS}

\section{H. SAMELSON}

Stanford University

Stanford, California

R. M. Blumenthal

University of Washington

Seattle, Washington 98105

\author{
*J. DugundJI \\ University of Southern California \\ Los Angeles, California 90007 \\ RICHARD ARENS \\ University of California \\ Los Angeles, California 90024
}

\section{ASSOCIATE EDITORS}
E. F. BECKENBACH
B. H. NeUManN
F. WolF
K. YosIDA

\section{SUPPORTING INSTITUTIONS}

UNIVERSITY OF BRITISH COLUMBIA
CALIFORNIA INSTITUTE OF TECHNOLOGY
UNIVERSITY OF CALIFORNIA
MONTANA STATE UNIVERSITY
UNIVERSITY OF NEVADA
NEW MEXICO STATE UNIVERSITY
OREGON STATE UNIVERSITY
UNIVERSITY OF OREGON
OSAKA UNIVERSITY
UNIVERSITY OF SOUTHERN CALIFORNIA

UNIVERSITY OF BRITISH COLUMBIA

UNIVERSITY OF CALIFORNIA

MONTANA STATE UNIVERSITY

NEW MEXICO STATE UNIVERSITY

OREGON STATE UNIVERSITY

OSAKA UNIVERSITY

UNIVERSITY OF SOUTHERN CALIFORNIA

\author{
STANFORD UNIVERSITY \\ UNIVERSITY OF TOKYO \\ UNIVERSITY OF UTAH \\ WASHINGTON STATE UNIVERSITY \\ UNIVERSITY OF WASHINGTON \\ AMERICAN MATHEMATICAL SOCIETY \\ CHEVRON RESEARCH CORPORATION \\ TRW SYSTEMS \\ NAVAL ORDNANCE TEST STATION
}




\section{Pacific Journal of Mathematics \\ Vol. 17, No. $1 \quad$ January, 1966}

Carlos Jorge Do Rego Borges, On stratifiable spaces ................ 1

Felix Earl Browder, Topological methods for non-linear elliptic equations of



Gustave Choquet, Harry Corson and Victor Klee, Exposed points of convex

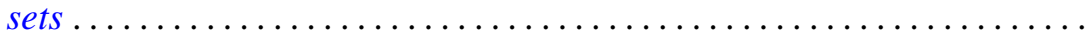

Phillip Emig, Remarks on the defect sum for a function meromorphic on an open Riemann surface ................................ 45

Ruth Goodman, A certain class of polynomials .................. 57

Sidney (Denny) L. Gulick, The bidual of a locally multiplicatively-convex



Eugene Carlyle Johnsen, Integral solutions to the incidence equation for finite projective plane cases of orders $n \equiv=2(\bmod 4) \ldots \ldots \ldots \ldots . .67$

Charles N. Kellogg, Centralizers and $H^{*}$-algebras .................. 121

Michael Lodato, On topologically induced generalized proximity relations. II .......................................... 131

P. H. Maserick, Half rings in linear spaces ..................... 137

Kathleen B O'Keefe, On a problem of J. F. Ritt .................... 149

Galen Lathrop Seever, Nonnegative projections on $C_{0}(X) \ldots \ldots \ldots \ldots$

Lawrence A. Shepp, Gaussian measures in function space ............ 167

Robert Charles Thompson, Classes of definite group matrices ........... 175 